\title{
Experimental Verification of Multidirectional Multiscroll Chaotic Attractors
}

\author{
Jinhu Lü, Member, IEEE, Simin Yu, Henry Leung, Member, IEEE, and Guanrong Chen, Fellow, IEEE
}

\begin{abstract}
A systematic methodology for circuit design is proposed for experimental verification of multidirectional multiscroll chaotic attractors, including one-directional (1-D) $n$-scroll, 2-D $n \times m$-grid scroll, and 3-D $n \times m \times l$-grid scroll chaotic attractors. Two typical cases are investigated in detail: the hysteresis and saturated multiscroll chaotic attractors. A simple blocking circuit diagram is designed for experimentally verifying 1-D $5 \sim 11$-scroll, 2-D $3 \times 5 \sim 11$-grid scroll, and 3-D $3 \times 3 \times 5 \sim 11$-grid scroll hysteresis chaotic attractors by manipulating the switchers. Moreover, a block circuitry is also designed for physically realizing 1-D 10, 12, 14-scroll, 2-D 10, 12, $14 \times 10$-grid scroll, and 3-D $10 \times 10 \times 10$-grid scroll saturated chaotic attractors via switching. In addition, one can easily realize chaotic attractors with a desired odd number of scrolls by slightly modifying the corresponding voltage saturated function series of the circuit, to produce for instance a 1-D 13-scroll saturated chaotic attractor. This is the first time in the literature to report an experimental verification of a 1-D 14-scroll, a 2 -D $14 \times 10$-grid scroll and a 3-D $10 \times 10 \times 10$-grid (totally 1000) scroll chaotic attractors. Only the 3-D case is reported in detail for simplicity of presentation. It is well known that hardware implementation of 1-D $n$-scroll with $n \geq 10,2$-D $n \times m$-grid scroll with $n, m \geq 10$, and 3-D $n \times m \times l$-grid scroll with $n, m, l \geq 10$ chaotic attractors is very difficult technically, signifying the novelty and significance of the achievements reported in this paper. Finally, this circuit design approach provides some principles and guidelines for hardware implementation of chaotic attractors with a multidirectional orientation and with a large number of scrolls, useful for future circuitry design and engineering applications.
\end{abstract}

Index Terms-Circuit implementation, hysteresis series, multidirectional chaotic attractor, multiscroll chaotic attractor, saturated series.

Manuscript received October 25, 2004; revised March 28, 2005. This work was supported in part by the National Natural Science Foundation of China under Grant 60304017, Grant 60572073, and Grant 20336040/B06, by the Natural Science Foundation of Guangdong Province under Grant 32469 and Grant 5001818, by the Science and Technology Program of Guangzhou City under Grant 2004J1-C0291, by the Hong Kong Research Grants Council under the CERG Grant CityU 1114/05E, and the Scientific Research Startup Special Foundation on Excellent Ph.D. Thesis and Presidential Award of Chinese Academy of Sciences. Recommended by Associate Editor Y. Nishio.

$\mathrm{J}$. Lü is with the Key Laboratory of Systems and Control, Institute of Systems Science, Academy of Mathematics and Systems Science, Chinese Academy of Sciences, Beijing 100080, China, and also with the Department of Ecology and Evolutionary Biology, Princeton University, Princeton, NJ 08540 USA (e-mail: jhlu@iss.ac.cn).

$\mathrm{S}$. Yu is with the College of Automation, Guangdong University of Technology, Guangzhou 510090, China.

H. Leung is with the Department of Electrical and Computer Engineering, University of Calgary, Calgary, AB T2N 1N4, Canada.

G. Chen is with the Department of Electronic Engineering, City University of Hong Kong, Hong Kong (e-mail: gchen@ee.cityu.edu.hk).

Digital Object Identifier 10.1109/TCSI.2005.854412

\section{INTRODUCTION}

$\mathbf{H}$ ARDWARE IMPLEMENTATION of reliable nonlinear circuits for generating various complex chaotic signals is a key issue for future applications of chaos-based information systems [1]-[4]. In particular, creating various complex multiscroll chaotic attractors by using some simple electronic devices is a topic of both theoretical and practical interests.

Research on generation of multiscroll chaotic attractors has been developing for more than a decade. There are many approaches reported in [1]-[43]. The so-called $n$-scroll attractors were first proposed by Suykens and Vandewalle [5]-[8]. Suykens and Chua [7] introduced the $n$-double scroll hypercubes in 1-D CNN. A family of hyperchaotic $n$-scroll attractors was also proposed by Yalcin et al. in [13]. These methods create multiscroll attractors by adding breakpoints in the nonlinear element of Chua's circuit or by using CNN with a piecewise-linear output function. A step function approach was introduced by Yalcin et al. in [10] for generating one-directional (1-D) $n$-scroll, two-directional (2-D) $n \times m$-grid scroll, and three-directional (3-D) $n \times m \times l$-grid scroll chaotic attractors. Tang et al. [2] proposed a sine-function method for creating $n$-scroll chaotic attractors. A nonlinear transconductor approach was also introduced for generating $n$-scroll attractors by Ozoguz et al. in [14]. Recently, Lü et al. [15], [16] proposed a switching manifold method for creating chaotic attractors with multiple-merged basins of attraction.

Hysteresis can also generate chaos easily [17]-[30]. Arena et al. [23] introduced a new realization for the Saito hysteresis chaos generator from a suitable connection of four generalized cellular neural network cells. Varrientos and Sánchez-Sinencio [24] presented a 4-D chaotic oscillator based on a differential hysteresis comparator. Saito et al. [18], [19] proposed some higher dimensional hysteresis chaos generators. Kataoka and Saito [25] reported a method to the synthesis of chaotic oscillators consisting of capacitors and two-port voltage-controlled current sources. Very recently, Lü et al. [29], [30] introduced a hysteresis series approach for creating 1-D $n$-scroll, 2-D $n \times$ $m$-grid scroll, and 3-D $n \times m \times l$-grid scroll chaotic attractors, with a rigorously mathematical proof for the chaotic behaviors. Moreover, a saturated function series method was also introduced by Lü et al. in [31], [32] for generating 1-D $n$-scroll, 2-D $n \times m$-grid scroll, and 3-D $n \times m \times l$-grid scroll attractors. All these multiscroll chaotic attractors have been verified by numerical simulations or theoretical proofs.

It has been realized that it is difficult to verify the multiscroll chaotic attractors by a physical electronic circuit. Arena et al. [43] firstly experimentally confirmed the $n$-double scroll chaotic attractor by a state-controlled cellular neural network 
(SC-CNN) based circuit. Yalcin et al. [37] physically realized the 3- and 5-scroll chaotic attractors in a generalized Chua's circuit. By using the scaling properties of the nonlinearity in a generalized Chua's circuit, Yalcin et al. [40] also experimentally confirmed a 6-scroll chaotic attractor. Until 2000, the largest number of scrolls that has been experimentally verified was only 6 , indicating the technical difficulty of the task. Circuit implementation of more than 6-scroll needs a larger dynamic range, requiring higher voltage supply and appropriate differential amplifiers or a convenient scaling of voltages [4]. Zhong et al. [1] proposed a systematical circuit design approach for physically realizing up to as many as ten scrolls visible on the oscilloscope. For the realization of multidirectional chaotic attractors, Yalcin et al. [10] experimentally verified the 2-D $3 \times 3$-grid scroll and 3-D $2 \times 2 \times 2$-grid scroll chaotic attractors. Recently, Yu et al. [33], [34] also physically realized the maximum 12-scroll chaotic attractors from a general jerk circuit. Last but not least, Lü and Chen [3] reviewed the main advances of multiscroll chaos generation in theories, methods, and applications. This, basically, is the state of the art in the field.

As is well known, it is much more difficult to physically realize a nonlinear resistor that has an appropriate characteristic with many segments [1]-[4]. Moreover, the realization of a nonlinear resistor with multisegments is a basis for hardware implementation of chaotic attractors with multidirectional orientation and with a large number of scrolls. One of the main obstacles is that the device must have a very wide dynamic range [3], [4], [33], [34], [40]; however, physical conditions always limit or even prohibit such circuit realization [1], [3].

It should be pointed out that hardware implementation of 1-D $n$-scroll with $n \geq 10$, 2-D $n \times m$-grid scroll with $n, m \geq 10$, and 3-D $n \times m \times l$-grid scroll with $n, m, l \geq 10$ chaotic attractors is very difficult due to the limitation of the dynamic ranges of the available physical circuit components [1]-[4], [33], [34], [37], [40]. Up to now, there are no significant results reported in the literature about physical realization of 2-D $n \times m$-grid scroll with $n, m \geq 10$ and 3-D $n \times m \times l$-grid scroll with $n, m, l \geq 10$ chaotic attractors. The main obstacles involve the realization of a nonlinear resistor with multisegments and the stability of a multiscroll circuit with a multidirectional orientation and with a large number of scrolls.

Considering the aforementioned difficulties, a systematic methodology for circuit design is proposed in this paper for experimentally verifying multidirectional multiscroll chaotic attractors. The design idea and approach provide a theoretical basis for physical realization of chaotic attractors with a multidirectional orientation and with a large number of scrolls. To explain the design idea, two typical cases are considered-the hysteresis and saturated multiscroll attractors-since hysteresis and saturated circuits are two different kinds of basic circuits. A simple block circuit diagram is introduced for experimentally verifying 1-D $5 \sim 11$-scroll, 2 -D $3 \times 5 \sim 11$-grid scroll, and 3 -D $3 \times 3 \times 5 \sim 11$-grid scroll hysteresis chaotic attractors via switching the switchers. Furthermore, a block circuitry is designed for physically realizing 1-D 10, 12, 14-scroll, 2-D $10,12,14 \times 10$-grid scroll, and 3-D $10 \times 10 \times 10$-grid scroll saturated chaotic attractors via switching. Also, one can easily realize chaotic attractors with a desired odd number of scrolls by slightly modifying the corresponding voltage saturated function series of the circuit, to produce for instance a 1-D 13-scroll saturated chaotic attractor. This is the first time in the literature to report the experimental confirmation of a 1-D 14-scroll, a 2 -D $14 \times 10$-grid scroll, and a 3 -D $10 \times 10 \times 10$-grid (totally 1000) scroll chaotic attractors. These experiments also show that realization of 3-D $n \times m \times l$-grid scroll with $n, m, l \geq 10$ chaotic attractor is significantly more difficult than that for the 1-D and 2-D settings. In this paper, only the 3-D case is discussed in detail, for simplicity of the presentation.

The rest of the paper is organized as follows. In Section II, the multidirectional hysteresis and saturated multiscroll chaotic systems are briefly introduced. Then, two novel block circuitries are constructed for hardware implementation of multidirectional hysteresis and saturated multiscroll chaotic attractors, in Sections III and IV, respectively. Conclusions are finally drawn in Section V.

\section{Multidirectional Multiscroll Chaotic AtTractors}

As is well known, hysteresis and saturated circuits are two different kinds of basic circuits. Generally speaking, hysteresis systems are discontinuous dynamical systems; however, saturated systems are continuous dynamical systems. In this paper, both hysteresis and saturated multiscroll chaotic systems are used as two representative examples of discontinuous and continuous dynamical systems, to explain how to design multidirectional multiscroll chaotic circuits. Moreover, the design ideas of hysteresis and saturated multiscroll chaotic circuits are also different from each other. This section briefly introduces such hysteresis and saturated chaotic systems.

\section{A. Hysteresis Multiscroll Chaotic Attractors}

Here, a hysteresis multiscroll chaotic system is introduced.

Recently, Lü et al. [30] proposed a three-dimensional hysteresis multiscroll chaotic system, described by

$$
\dot{\mathbf{X}}=\mathbf{A} \mathbf{X}+\mathbf{B} \boldsymbol{\theta}(\mathbf{X})
$$

where $\mathbf{X}=(x, y, z)^{T}$ is the state vector, $\mathbf{B}=-\mathbf{A}$, and

$$
\mathbf{A}=\left(\begin{array}{ccc}
0 & 1 & 0 \\
0 & 0 & 1 \\
-a & -b & -c
\end{array}\right)
$$

There are three different cases to consider, as follows:

1) 1-D hysteresis $n$-scroll attractors

$$
\boldsymbol{\theta}(\mathbf{X})=\left(\begin{array}{c}
h\left(x, p_{1}, q_{1}\right) \\
0 \\
0
\end{array}\right)
$$

where the hysteresis series function $h\left(x, p_{1}, q_{1}\right)$ is defined by

$$
h\left(x, p_{1}, q_{1}\right)= \begin{cases}-p_{1}, & \text { if } x<-p_{1}+1 \\ i, & \text { if } i=1<x<i+1 \\ q_{1}, & \text { if } x>q_{1}-1 . \ldots, q_{1}-1\end{cases}
$$


2) 2-D hysteresis $n \times m$-grid scroll attractors

$$
\boldsymbol{\theta}(\mathbf{X})=\left(\begin{array}{c}
h\left(x, p_{1}, q_{1}\right) \\
h\left(y, p_{2}, q_{2}\right) \\
0
\end{array}\right)
$$

where the hysteresis series functions $h\left(x, p_{1}, q_{1}\right)$ and $h\left(y, p_{2}, q_{2}\right)$ are defined by (4).

3) 3-D hysteresis $n \times m \times l$-grid scroll attractors

$$
\boldsymbol{\theta}(\mathbf{X})=\left(\begin{array}{l}
h\left(x, p_{1}, q_{1}\right) \\
h\left(y, p_{2}, q_{2}\right) \\
h\left(z, p_{3}, q_{3}\right)
\end{array}\right)
$$

where the hysteresis series functions $h\left(x, p_{1}, q_{1}\right)$, $h\left(y, p_{2}, q_{2}\right)$, and $h\left(z, p_{3}, q_{3}\right)$ are defined by (4).

Note that (1) with controller (3) can generate a 1-D $\left(p_{1}+\right.$ $\left.q_{1}+1\right)$-scroll chaotic attractor for some suitable parameters $a$, $b, c$; (1) with controller (5) can create a 2-D $\left(p_{1}+q_{1}+1\right) \times$ $\left(p_{2}+q_{2}+1\right)$-grid scroll chaotic attractor for some suitable parameters $a, b, c$; (1) with controller (6) can generate a 3-D $\left(p_{1}+q_{1}+1\right) \times\left(p_{2}+q_{2}+1\right) \times\left(p_{3}+q_{3}+1\right)$-grid scroll chaotic attractor for some suitable parameters $a, b, c$. For example, when $a=0.8, b=0.72, c=0.6, p_{1}=q_{1}=3$, (1) with (3) has a 1-D 7-scroll chaotic attractor as shown in [30, Fig. 2]; when $a=0.8$, $b=0.7, c=0.6, p_{1}=q_{1}=2, p_{2}=q_{2}=3$, (1) with (5) has a 2-D $5 \times 7$-grid scroll chaotic attractor as shown in [30, Fig. 3]; when $a=0.8, b=0.72, c=0.66, p_{1}=q_{1}=2, p_{2}=3$, $q_{2}=4, p_{3}=q_{3}=1$, (1) with (6) has a 3 -D $5 \times 8 \times 3$-grid scroll chaotic attractor, as shown in [30, Fig. 4].

Moreover, Lü et al. [30] constructed a two-dimensional Poincaré return map to verify the chaotic behaviors of the generated multidirectional hysteresis multiscroll chaotic attractors via a rigorous theoretical approach. To that end, however, physically experimental observations are still lacking. To bridge the gap, a systematic method is presented here for generating hysteresis multiscroll chaotic attractors by a physical electronic circuit in Section III.

\section{B. Saturated Multiscroll Chaotic Attractors}

Very recently, Lü et al. [31], [32] proposed a three-dimensional saturated multiscroll chaotic system, which is described by (1) with

$$
\mathbf{A}=\left(\begin{array}{ccc}
0 & 1 & 0 \\
0 & 0 & 1 \\
-a & -b & -c
\end{array}\right), \mathbf{B}=\left(\begin{array}{ccc}
0 & -\frac{d_{2}}{b} & 0 \\
0 & 0 & -\frac{d_{3}}{c} \\
d_{1} & d_{2} & d_{3}
\end{array}\right)
$$

The attractors generated by this system can be classified into three categories, as follows:

1) 1-D saturated $n$-scroll chaotic attractors

$$
\boldsymbol{\theta}(\mathbf{X})=\left(\begin{array}{c}
f\left(x ; k_{1}, h_{1}, p_{1}, q_{1}\right) \\
0 \\
0
\end{array}\right)
$$

where the saturated function series $f\left(x ; k_{1}, h_{1}, p_{1}, q_{1}\right)$ is defined by

$$
\begin{aligned}
& f\left(x ; k_{1}, h_{1}, p_{1}, q_{1}\right) \\
& \quad= \begin{cases}\left(2 q_{1}+1\right) k_{1}, & \text { if } x>q_{1} h_{1}+1 \\
k_{1}\left(x-i h_{1}\right)+2 i k_{1}, & \text { if }\left|x-i h_{1}\right| \leq 1 \\
& -p_{1} \leq i \leq q_{1} \\
(2 i+1) k_{1}, & \text { if } 1<x-i h_{1}<h_{1}-1 \\
-\left(2 p_{1}+1\right) k_{1}, & \text { if } x<-p_{1} \leq i \leq q_{1}-1\end{cases}
\end{aligned}
$$

2) 2-D saturated $n \times m$-grid scroll chaotic attractors:

$$
\boldsymbol{\theta}(\mathbf{X})=\left(\begin{array}{c}
f\left(x ; k_{1}, h_{1}, p_{1}, q_{1}\right) \\
f\left(y ; k_{2}, h_{2}, p_{2}, q_{2}\right) \\
0
\end{array}\right)
$$

where $f\left(x ; k_{1}, h_{1}, p_{1}, q_{1}\right)$ and $f\left(y ; k_{2}, h_{2}, p_{2}, q_{2}\right)$ are similarly defined by (9).

3) 3-D saturated $n \times m \times l$-grid scroll chaotic attractors

$$
\boldsymbol{\theta}(\mathbf{X})=\left(\begin{array}{l}
f\left(x ; k_{1}, h_{1}, p_{1}, q_{1}\right) \\
f\left(y ; k_{2}, h_{2}, p_{2}, q_{2}\right) \\
f\left(z ; k_{3}, h_{3}, p_{3}, q_{3}\right)
\end{array}\right)
$$

where $f\left(x ; k_{1}, h_{1}, p_{1}, q_{1}\right), f\left(y ; k_{2}, h_{2}, p_{2}, q_{2}\right)$, and $f\left(z ; k_{3}, h_{3}, p_{3}, q_{3}\right)$ are similarly defined by (9).

Moreover, (1) with (7) and saturated controllers (8), (10), and (11), respectively, can generate 1-D $\left(p_{1}+q_{1}+2\right)$-scroll, 2-D $\left(p_{1}+q_{1}+2\right) \times\left(p_{2}+q_{2}+2\right)$-grid scroll, and 3-D $\left(p_{1}+q_{1}+2\right) \times$ $\left(p_{2}+q_{2}+2\right) \times\left(p_{3}+q_{3}+2\right)$-grid scroll chaotic attractors for some suitable system parameters. For example, (1) with (7) and (8) generates a 1-D 6-scroll chaotic attractor as shown in [31, Fig. 6] for $a=b=c=d_{1}=0.7, k_{1}=9, h_{1}=18, p_{1}=2, q_{1}=$ 2 ; (1) with (7) and (10) creates a 2-D $6 \times 6$-grid scroll chaotic attractor as shown in [31, Fig. 7] for $a=b=c=d_{1}=d_{2}=$ $0.7, k_{1}=k_{2}=50, h_{1}=h_{2}=100, p_{1}=q_{1}=p_{2}=q_{2}=2$ and (1) with (7) and (11) generates a 3-D $6 \times 6 \times 6$-grid scroll chaotic attractor as shown in [31, Fig. 8] for $a=d_{1}=0.7$, $b=c=d_{2}=d_{3}=0.8, k_{1}=100, h_{1}=200, k_{2}=k_{3}=40$, $h_{2}=h_{3}=80, p_{1}=p_{2}=p_{3}=q_{1}=q_{2}=q_{3}=2$.

It is worth noting that the dynamic range of the 3-D $6 \times 6 \times 6$-grid scroll chaotic attractor shown in [31, Fig. 8] is $x \in[-800,800], y \in[-500,500], z \in[-500,500]$. Similarly, the dynamic ranges of other multiscroll chaotic attractors are within the same quantity level $\left(O\left(10^{2}\right)\right)$. However, the dynamic ranges for most analog electronic devices in laboratories are only [-13.5 V, $13.5 \mathrm{~V}]$, with power supplies \pm $15 \mathrm{~V}$ [33]-[36]. To experimentally verify the above saturated multiscroll chaotic attractors, one has to rescale the dynamic ranges of the multiscroll chaotic attractors. For this purpose, one needs to rescale the dynamic range of the saturated function series (9). To do so, the definition of saturated function series (9) is redefined as follows:

$$
\begin{aligned}
& f\left(x ; e_{1}, h_{1}, k_{1}, p_{1}, q_{1}\right) \\
& \quad= \begin{cases}\left(2 q_{1}+1\right) k_{1}, & \text { if } x>q_{1} h_{1}+e_{1} \\
\frac{k_{1}}{e_{1}}\left(x-i h_{1}\right)+2 i k_{1}, & \text { if } \quad-p_{1} \leq i \leq h_{1} \mid \leq e_{1} \\
(2 i+1) k_{1}, & \text { if } e_{1}<x-i h_{1}<h_{1}-e_{1} \\
-\left(2 p_{1}+1\right) k_{1}, & \text { if } x<-p_{1} \leq q_{1}-1\end{cases} \\
&
\end{aligned}
$$




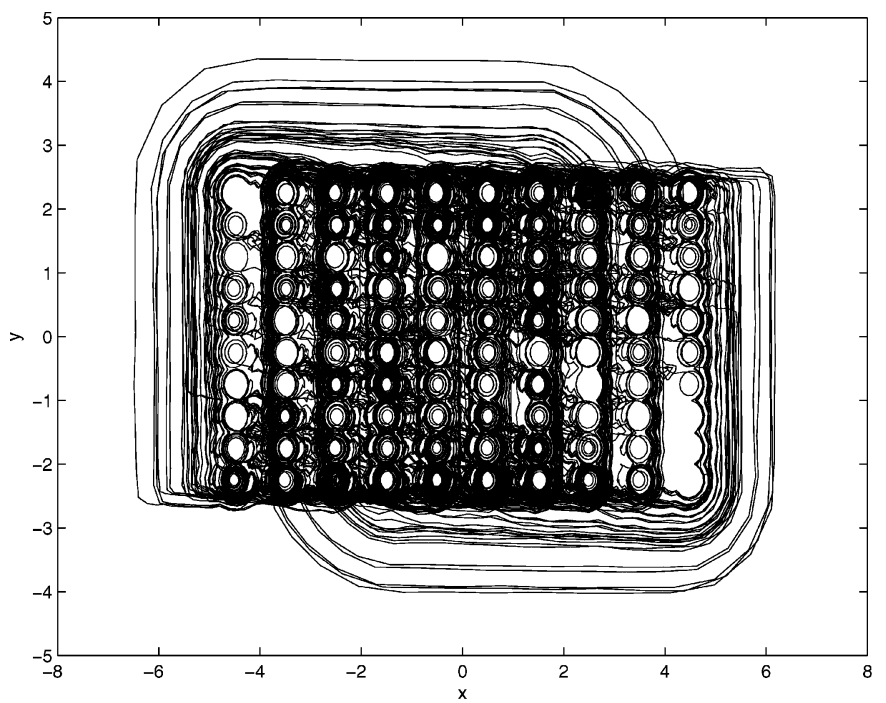

(a)

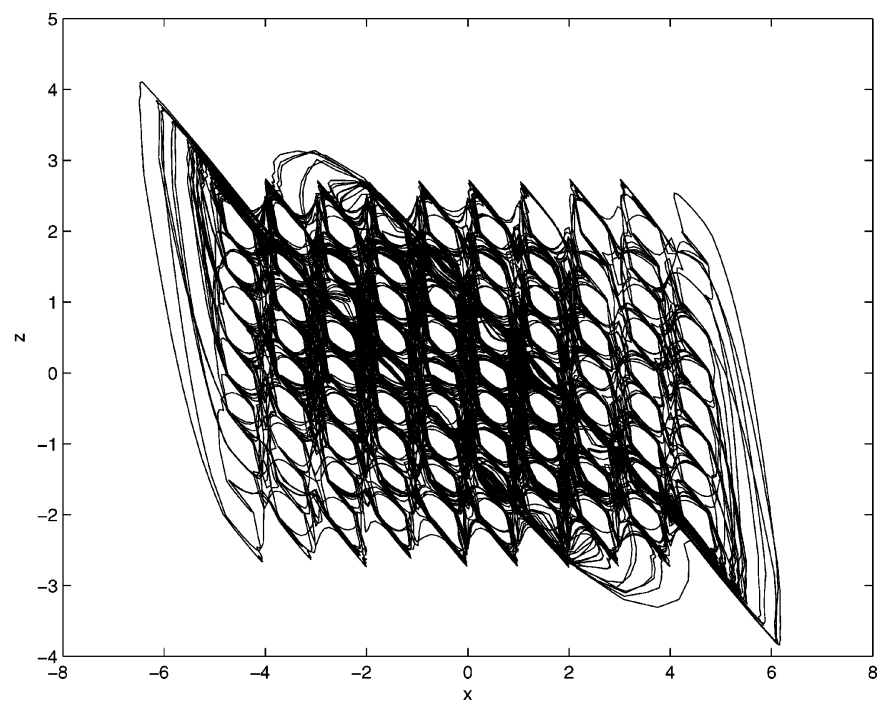

(b)

Fig. 1. $10 \times 10 \times 10$-grid scroll saturated chaotic attractor. (a) $x-y$ plane. (b) $x-z$ plane.

When $a=b=c=d_{1}=d_{2}=d_{3}=0.8, e_{1}=0.005$, $e_{2}=e_{3}=0.0025, h_{1}=1, h_{2}=h_{3}=0.5, k_{1}=0.5$, $k_{2}=k_{3}=0.25, p_{1}=p_{2}=p_{3}=q_{1}=q_{2}=q_{3}=4$, (1) with (7), (11) and (12) has a 3-D $10 \times 10 \times 10$-grid scroll chaotic attractor as shown in Fig. 1. Fig. 1(a) and (b) shows the $x-y$ and $x-z$ plane projections, respectively. Clearly, the dynamic range of above 3 -D $10 \times 10 \times 10$-grid scroll chaotic attractor is: $x \in[-8,8], y \in[-5,5], z \in[-5,5]$. In our experiments, the voltages of the positive and negative power supplies are $\pm 15 \mathrm{~V}$ and the outputs of the saturated voltages are $\pm 13.5 \mathrm{~V}$. By taking into account the precision in calculation, the maximum dynamic ranges of all operational amplifiers are $[-10 \mathrm{~V}, 10 \mathrm{~V}]$.

Because (1) with (7) is continuous in $\mathbf{R}^{3}$, it has some very good analytical properties as compared to the corresponding step multiscroll system studied in [10] and hysteresis multiscroll system (1) with (2) are discontinuous in all switching points. Although Lü et al. [31] have designed a two-dimensional Poincaré return map to verify the chaotic behaviors of the created multidirectional saturated multiscroll chaotic attractors via a rigorous theoretical approach, experimental verification of these results is still lacking. In Section IV, to bridge this gap, a systematic approach is proposed for generating saturated multiscroll chaotic attractors by a physical electronic circuit.

\section{Design OF MulTidiRECTIONAl Hysteresis MULTISCROLL CIRCUITS}

In this section, a novel blocking circuit diagram is constructed for experimental verification of multidirectional hysteresis multiscroll chaotic attractors, including 1-D $n$-scroll, 2-D $n \times m$-grid scroll, and 3-D $n \times m \times l$-grid scroll chaotic attractors.

\section{A. Multidirectional Hysteresis Multiscroll Circuits and Experimental Observations}

In this subsection, a circuit diagram is introduced for generating multidirectional hysteresis multiscroll chaotic attractors, with experimental observations reported.

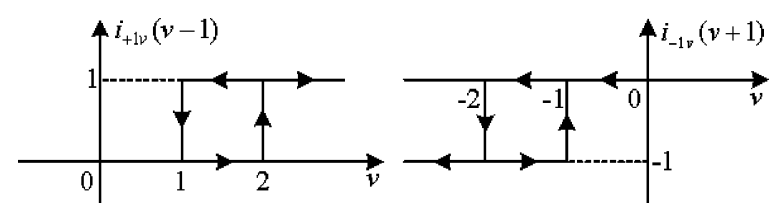

Fig. 2. Hysteresis currents $i_{+v}(v-1)$ and $i_{-v}(v+1)$.

A hysteresis current function is described by

$$
i_{+n v}\left(v-n v_{0}\right)= \begin{cases}0, & \text { if } v-n v_{0}<1 \\ 1, & \text { if } v-n v_{0}>0\end{cases}
$$

or

$$
i_{-n v}\left(v+n v_{0}\right)= \begin{cases}0, & \text { if } v+n v_{0}>-1 \\ -1, & \text { if } v+n v_{0}<0\end{cases}
$$

where $\left(v, v_{0}\right) \in\left\{\left(x, x_{0}\right),\left(y, y_{0}\right),\left(z, z_{0}\right)\right\}, x_{0}=y_{0}=z_{0}=$ $1 \mathrm{~V}$, and $n$ is a nonnegative integer. Fig. 2 shows the hysteresis current function for $n=1$.

In the following, the basic hysteresis current functions (13) and (14) are applied to construct the hysteresis series function generator (4) in the proposed circuit design.

According to (1)-(6) and (13)-(14), we design a circuit diagram for generating multidirectional hysteresis multiscroll chaotic attractors, as shown in Fig. 3. This diagram includes four different function parts: $N_{1}, N_{2}, N_{3}, N_{4}$. More precisely, $N_{1}$ consists of three integrators and two subtractor amplifiers; $N_{2}$ is the generator for hysteresis current series $i_{h}(x) ; N_{3}$ includes the generator for hysteresis current series $I_{h}(y)$ and the generator for hysteresis voltage series $V_{h}(y) ; N_{4}$ consists of the generator for hysteresis current series $I_{h}(z)$ and the generator for hysteresis voltage series $V_{h}(z)$. Fig. 4 shows the circuit diagram of the hysteresis series generator $N_{2}$. The switches $K 1, K 2, K 3, K 4$ in Fig. 3 are used to control the hysteresis series $V_{h}(y), V_{h}(z), I_{h}(y)$, and $I_{h}(z)$, respectively. Therefore, the switches $K 1, K 2, K 3, K 4$ in Fig. 3 can control the number of directions for the hysteresis multiscroll chaotic attractors, as summarized in Table I. On the other hand, the 


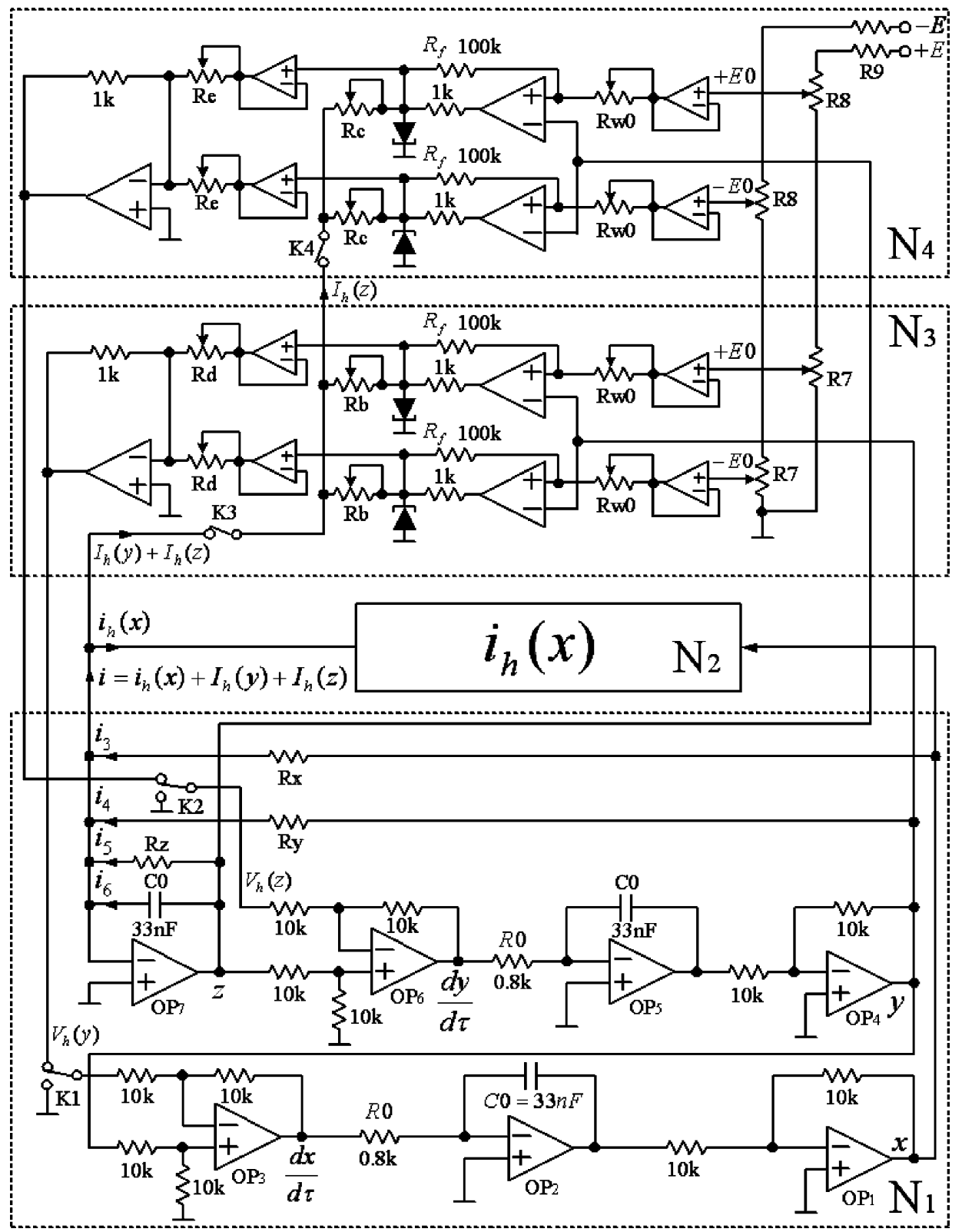

Fig. 3. Circuit diagram of multidirectional hysteresis multiscroll chaotic circuits.

TABLE I

ON-OFF OF SWITCHES $K 1 \sim K 4$ AND THE NUMBER OF DIRECTIONS FOR THE MULTISCROLL ATTRACTORS

\begin{tabular}{c|c|c|c|c}
\hline$K 1$ & $K 2$ & $K 3$ & $K 4$ & Number of directions \\
\hline off & off & off & off & 1 \\
\hline on & off & on & off & 2 \\
\hline on & on & on & on & 3 \\
\hline
\end{tabular}

switches $K_{ \pm n}(n=2,3,4)$ in Fig. 4 are used to control the number of scrolls in the $x$-direction, as summarized in Table II. Moreover, all operational amplifiers in Figs. 3 and 4 are TL082 and the supply voltage of the electrical source is $E=15 \mathrm{~V}$.

According to Table I, when the switches $K 1, K 2, K 3, K 4$ are switched off, the circuitry shown in Fig. 3 can generate 1-D $5 \sim 11$-scroll chaotic attractors by switching the switches $K_{ \pm n}$ $(n=2,3,4)$ shown in Fig. 4 and Table II. Fig. 5 shows the ex-
TABLE II

ON-OFF OF SWITCHES $K_{ \pm n}(n=2,3,4)$ AND THE NuMBER OF SCROLLS $N$

\begin{tabular}{c|c|c|c|c|c|c}
\hline$K_{+2}$ & $K_{-2}$ & $K_{+3}$ & $K_{-3}$ & $K_{+4}$ & $K_{-4}$ & $N$ \\
\hline off & off & off & off & off & off & 5 \\
\hline on & off & off & off & off & off & 6 \\
\hline on & on & off & off & off & off & 7 \\
\hline on & on & on & off & off & off & 8 \\
\hline on & on & on & on & off & off & 9 \\
\hline on & on & on & on & on & off & 10 \\
\hline on & on & on & on & on & on & 11 \\
\hline
\end{tabular}

perimental observations of 1-D 10,11-scroll chaotic attractors in the $x-y$ plane.

As seen from Table I, when the switches $K 1, K 3$ are switched on and the switches $K 2, K 4$ are switched off, the circuitry shown in Fig. 3 can create 2-D $3 \times 5 \sim 11$-grid scroll chaotic attractors 


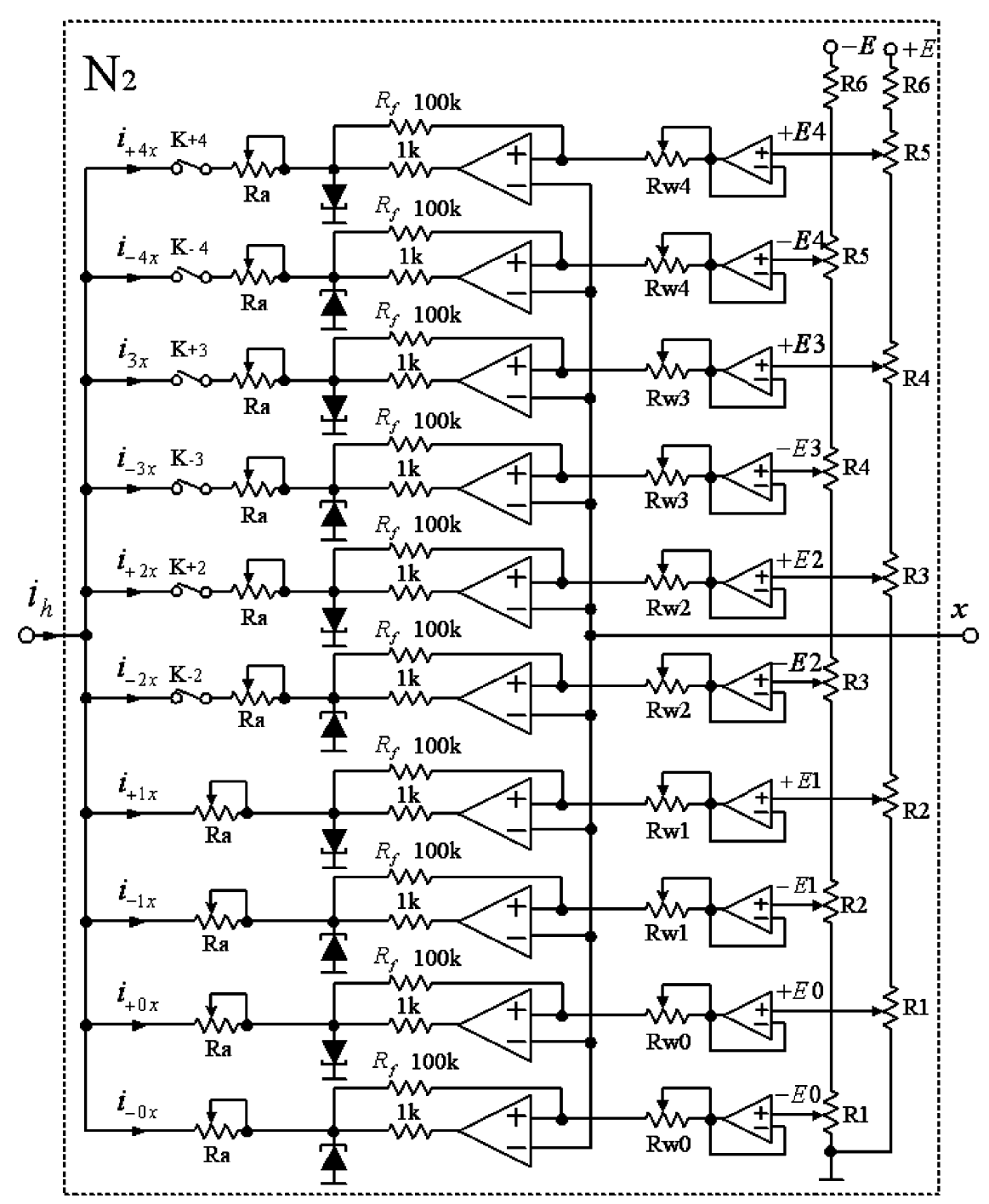

Fig. 4. Circuit diagram of the hysteresis series generator.

by switching the switches $K_{ \pm n}(n=2,3,4)$ shown in Fig. 4 and Table II. Fig. 6 shows the experimental observations of 2-D $3 \times 10,11$-grid scroll chaotic attractors in the $x-y$ plane.

According to Table I, when the switches $K 1, K 2, K 3, K 4$ are switched on, the circuitry shown in Fig. 3 can generate $3-D$ $3 \times 3 \times 5 \sim 11$-grid scroll chaotic attractors by switching the switches $K_{ \pm n}(n=2,3,4)$ shown in Fig. 4 and Table II. Fig. 7 shows the experimental observations of 3 -D $3 \times 3 \times 10,11$-grid scroll chaotic attractors in the $x-y$ plane. Fig. 8 shows the experimental observations of 3 -D $3 \times 3 \times 10,11$-grid scroll chaotic attractors in the $x-z$ plane.

\section{B. Dynamic Equations of Hysteresis Multiscroll Circuits}

In this section, the dynamic equations of the designed hysteresis multiscroll circuits are rigorously derived from the circuit diagram Fig. 3.

Define

$$
K_{ \pm n}= \begin{cases}1, & \text { if } K_{ \pm n} \text { is switched on } \\ 0, & \text { if } K_{ \pm n} \text { is switched off. }\end{cases}
$$

According to (13)-(14) and based on the circuit diagrams shown in Figs. 3 and 4, one obtains the total hysteresis current $i_{h}(x)$ of the generator $N_{2}$ as follows:

$$
\begin{aligned}
i_{h}(x)= & \frac{V_{S}}{R_{a}}\left[\sum_{n=0}^{1} i_{-n x}\left(x+n x_{0}\right)+\sum_{n=0}^{1} i_{+n x}\left(x-n x_{0}\right)\right. \\
& \left.+\sum_{n=2}^{4} K_{-n} i_{-n x}\left(x+n x_{0}\right)+\sum_{n=2}^{4} K_{+n} i_{+n x}\left(x-n x_{0}\right)\right] \\
= & \sum_{n=0}^{1} i_{-n x}\left(x+n x_{0}\right)+\sum_{n=0}^{1} i_{+n x}\left(x-n x_{0}\right) \\
& +\sum_{n=2}^{4} K_{-n} i_{-n x}\left(x+n x_{0}\right)+\sum_{n=2}^{4} K_{+n} i_{+n x}\left(x-n x_{0}\right)
\end{aligned}
$$

where $x_{0}=1 \mathrm{~V}, n x_{0}(0 \leq n \leq 4)$ are the shift voltages of the hysteresis current functions, $\bar{V}_{S}=7.5 \mathrm{~V}$ is the manostatic value of the zener diode, $R_{a}=7.5 \mathrm{k} \Omega$ is the voltage-current conversion resistor, and the unit of $i_{h}(x)$ is milliampere. 


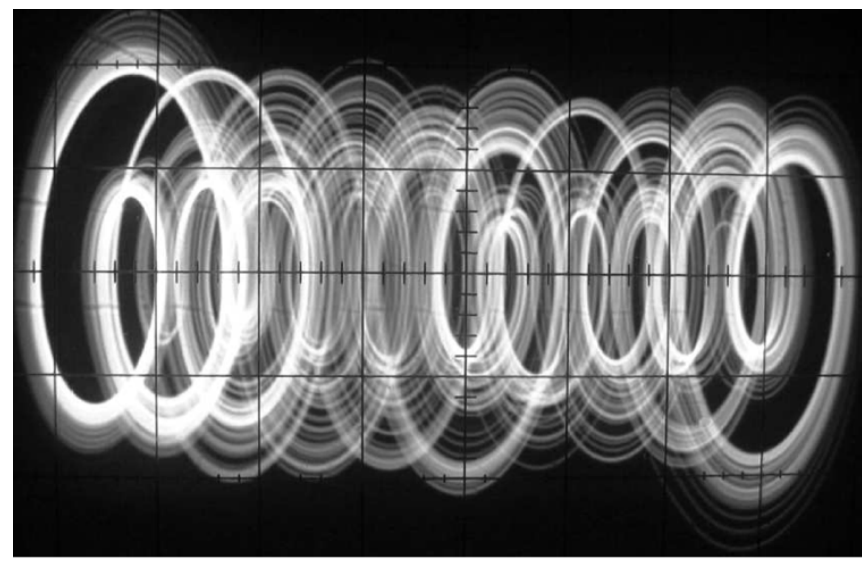

(a)

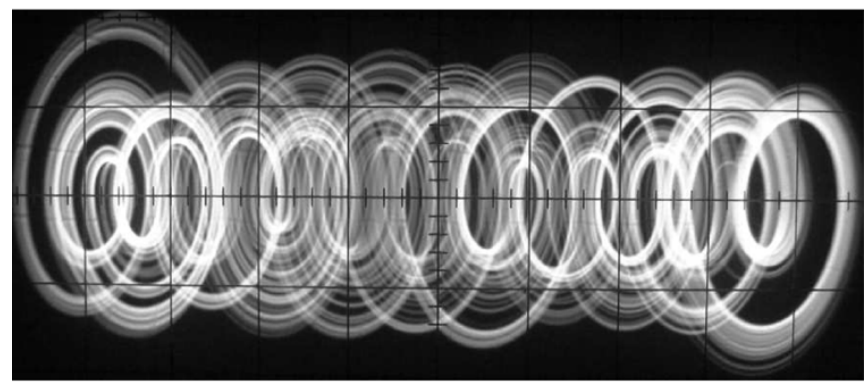

(b)

Fig. 5. Experimental observations of 1-D hysteresis multiscroll chaotic attractors in the $x-y$ plane. From up to down: (a) 10-scroll, where $x=1.3 \mathrm{~V} / \operatorname{div}, y=0.75 \mathrm{~V} /$ div. (b) 11 -scroll, where $x=1.3 \mathrm{~V} / \mathrm{div}$, $y=0.75 \mathrm{~V} / \mathrm{div}$.

One also obtains the total hysteresis current $I_{h}(y)$ of $N_{3}$ as follows:

$I_{h}(y)=\frac{V_{S}}{R_{b}}\left[\sum_{n=0}^{1} i_{-n y}\left(y+n y_{0}\right)+\sum_{n=0}^{1} i_{+n y}\left(y-n y_{0}\right)\right]=0.9 i_{h}(y)$

where $R_{b}=8.3 \mathrm{k} \Omega, y_{0}=1 \mathrm{~V}$, and the unit of $I_{h}(y)$ is milliampere.

Similarly, one gets the total hysteresis current $I_{h}(z)$ of $N_{4}$ as follows:

$I_{h}(z)=\frac{V_{S}}{R_{c}}\left[\sum_{n=0}^{1} i_{-n z}\left(z+n z_{0}\right)+\sum_{n=0}^{1} i_{+n z}\left(z-n z_{0}\right)\right]=0.825 i_{h}(z)$

where $R_{c}=9.1 \mathrm{k} \Omega, z_{0}=1 \mathrm{~V}$, and the unit of $I_{h}(z)$ is milliampere.

Therefore, the total hysteresis current of $N 2$ and $N 3$ is

$$
i=i_{h}(x)+I_{h}(y)=i_{h}(x)+0.9 i_{h}(y)
$$

and the total hysteresis current of $N 2, N 3, N 4$ is

$$
i=i_{h}(x)+I_{h}(y)+I_{h}(z)=i_{h}(x)+0.9 i_{h}(y)+0.825 i_{h}(z) .
$$

According to (16)-(18), the input voltages $E_{n}(0 \leq n \leq 4)$ of the operation amplifiers in $N_{2}, N_{3}, N_{4}$ can be obtained as follows:

$$
E_{n}=\frac{R_{f}+R_{w n}}{2 R_{f}}\left(2 n v_{0}+v_{0}+V_{S}\right)-\frac{V_{S}}{2}
$$

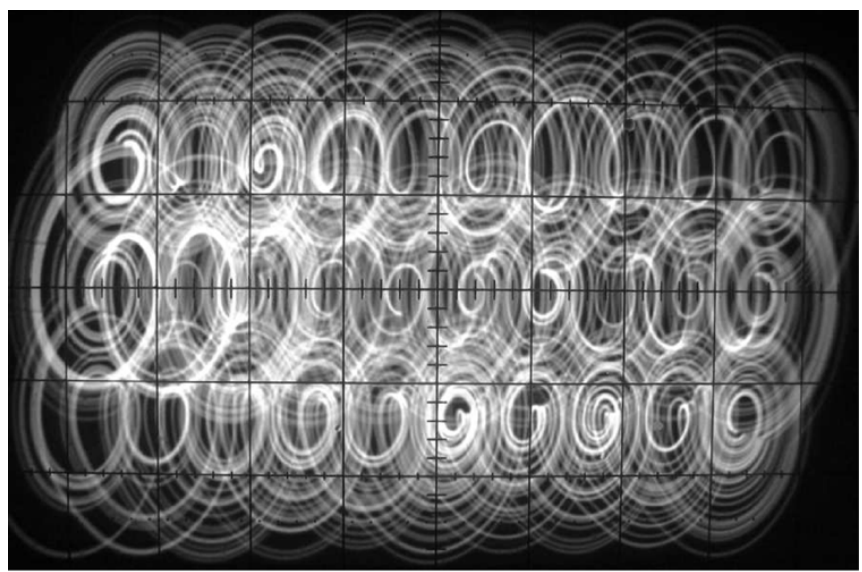

(a)

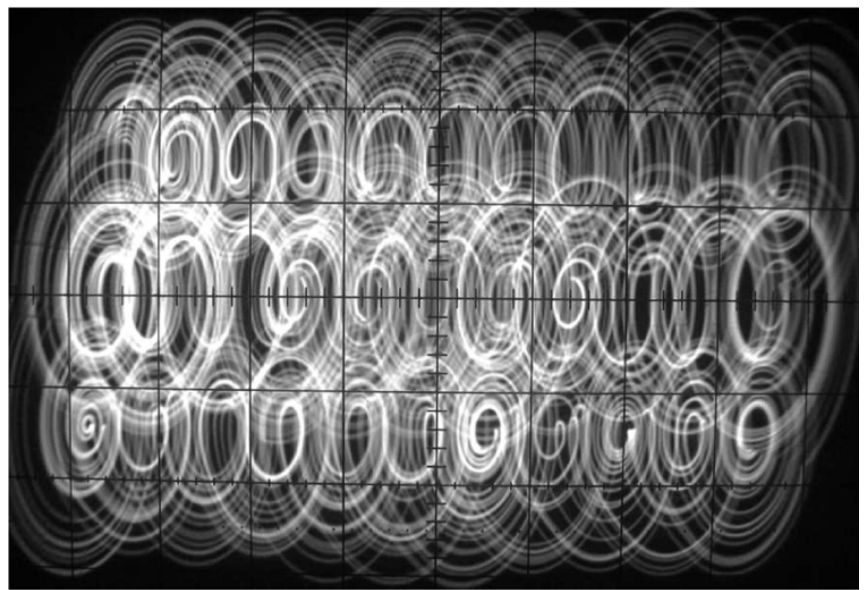

(b)

Fig. 6. Experimental observations of 2-D hysteresis multiscroll chaotic attractors in the $x-y$ plane. From up to down: (a) $3 \times 10$-grid scroll, where $x=1.3 \mathrm{~V} /$ div, $y=0.8 \mathrm{~V} /$ div. (b) $3 \times 11$-grid scroll, where $x=1.44 \mathrm{~V} /$ div,$y=0.8 \mathrm{~V} /$ div.

where $R_{w n}=9 \mathrm{k} \Omega(0 \leq n \leq 4), R_{f}=100 \mathrm{k} \Omega, V_{S}=7.5 \mathrm{~V}$, and $v_{0} \in\left\{x_{0}, y_{0}, z_{0}\right\}$ with $x_{0}=y_{0}=z_{0}=1 \mathrm{~V}$.

According to the recursive formulas (21), one can calculate the input voltages $E_{n}(0 \leq n \leq 4)$ of the operation amplifiers as follows:

$$
\begin{aligned}
& E_{0}=0.8825, E_{1}=1.9725, E_{2}=3.0625 \\
& E_{3}=4.1525, E_{4}=5.2425
\end{aligned}
$$

where the units of all voltages $E_{n}(0 \leq n \leq 4)$ are volt. The real measurement values of $E_{n}(0 \leq n \leq 4)$ in the circuit experiment may have a small departure from the theoretically calculated values of (22), due to the discreteness of the real circuit parameters and the measurement errors. The differences can be corrected via a small adjustment of the resistors $R_{w 0} \sim R_{w 4}$ and $R_{1} \sim R_{9}$ in the circuit implementation.

Because $E_{n}<E(0 \leq n \leq 4)$, from (21), the manostatic value $V_{S}$ of the zener diode should satisfy the following condition

$$
V_{S}<\frac{2 R_{f}}{R_{w n}}\left[E-\frac{R_{f}+R_{w n}}{2 R_{f}}(2 n+1) v_{0}\right]
$$

where $0 \leq n \leq 4$ 


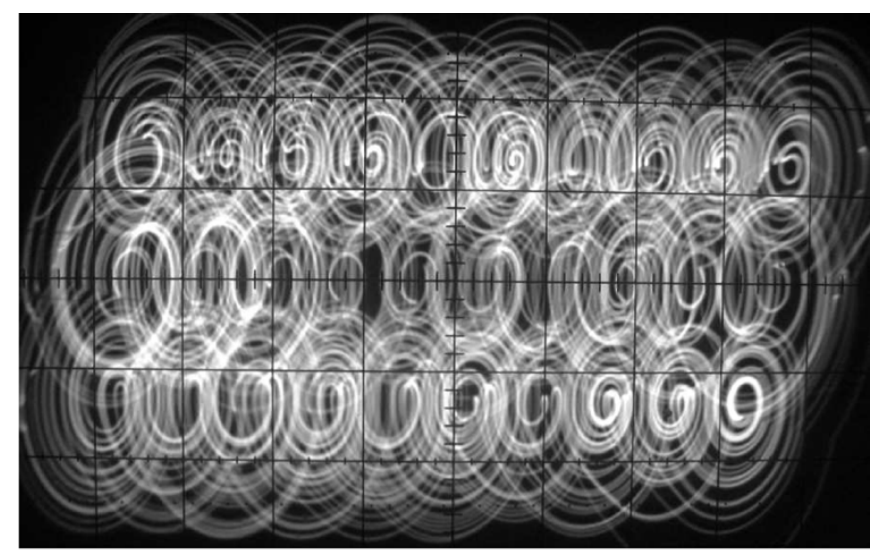

(a)

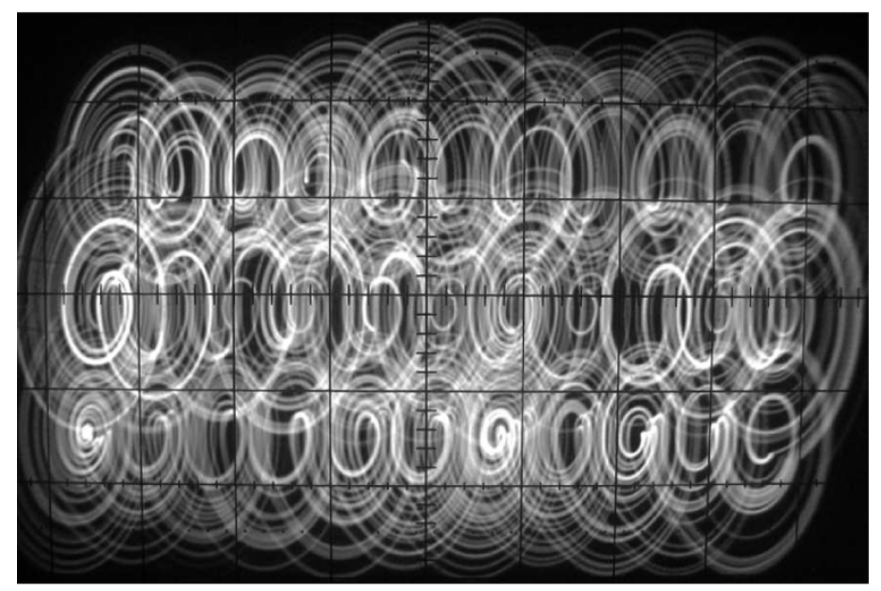

(b)

Fig. 7. Experimental observations of 3-D hysteresis multiscroll chaotic attractors in the $x-y$ plane. From up to down: (a) $3 \times 3 \times 10$-grid scroll, where $x=1.3 \mathrm{~V} /$ div, $y=0.8 \mathrm{~V} /$ div. (b) $3 \times 3 \times 11$-grid scroll, where $x=1.44 \mathrm{~V} /$ div,$y=0.8 \mathrm{~V} /$ div.

Remark 1: It is well known that the number $N$ of scrolls depends on the voltage $E$ of the electrical source and the displacement $v_{0}$ of the hysteresis function. In theory, the maximum number $N_{\max }$ of scrolls should satisfy $N_{\max }<2\left(E / v_{0}\right)$. Let $E=15 \mathrm{~V}, v_{0}=1 \mathrm{~V}$, then $N_{\max }<2\left(E / v_{0}\right)=30$. However, because of the limitation of the dynamic range of operational amplifier and the parameter discretization of other electronic devices, the maximum number of scrolls that can be realized in the practical circuit is far less than the above theoretically critical value. In fact, it is very difficult to physically realize more than 10 scrolls from technical view.

One can rigorously derive the dynamic equations of hysteresis multiscroll circuits from Fig. 3. In the following, only the 3-D setting is described in detail, since the 1-D and 2-D settings are special cases of the $3-\mathrm{D}$ setting and they can be similarly discussed.

Assume that $K 1, K 2, K 3, K 4$ in Fig. 3 are switched on. Based on the circuit diagram shown in Fig. 3, using the node analysis approach from circuit theory, one can obtain the circuit equation on the noninverting terminal of the operational amplifier $\mathrm{OP}_{7}$, as follows:

$$
i_{3}+i_{4}+i_{5}+i_{6}=i_{h}(x)+0.9 i_{h}(y)+0.825 i_{h}(z) .
$$

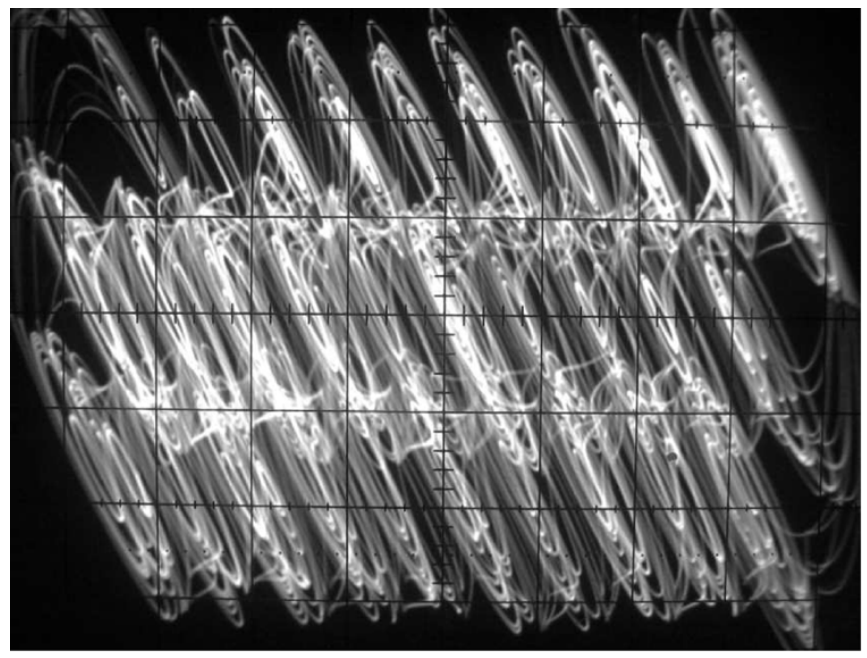

(a)

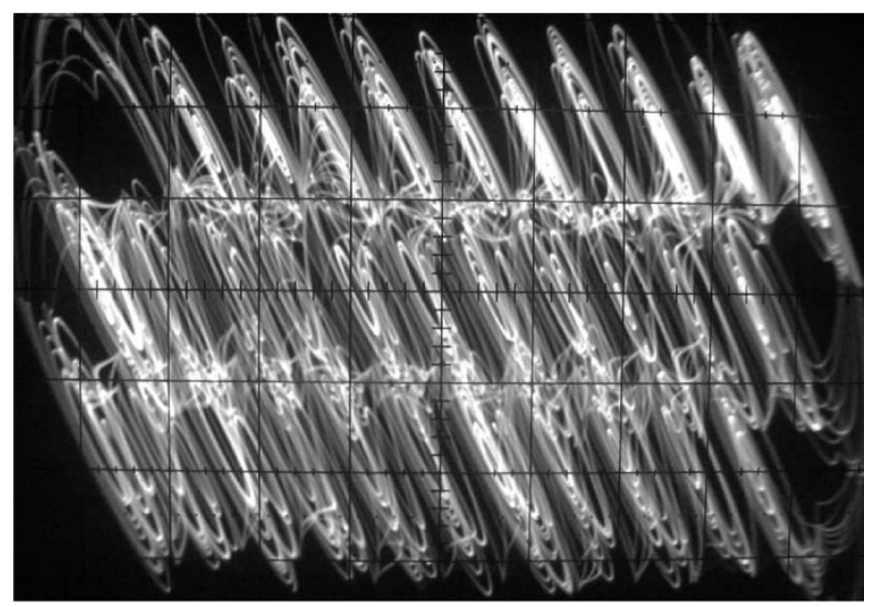

(b)

Fig. 8. Experimental observations of 3-D hysteresis multiscroll chaotic attractors in the $x-z$ plane. From up to down: (a) $3 \times 3 \times 10$-grid scroll, where $x=1.3 \mathrm{~V} /$ div, $z=0.8 \mathrm{~V} /$ div. (b) $3 \times 3 \times 11$-grid scroll, where $x=1.44 \mathrm{~V} / \mathrm{div}, z=0.8 \mathrm{~V} / \mathrm{div}$

where $i_{h}(x), 0.9 i_{h}(y)$, and $0.825 i_{h}(z)$ are defined by (16)-(18), respectively, and $i_{3}, i_{4}, i_{5}, i_{6}$ are also defined by

$$
\left\{\begin{array}{l}
i_{3}=\frac{x}{R_{x}} \\
i_{4}=\frac{y^{2}}{R_{y}} \\
i_{5}=\frac{z}{R_{z}} \\
i_{6}=\frac{C_{0} d z}{d t}
\end{array} .\right.
$$

Multiplying $R_{0}$ to both sides of (23), and using (23)-(24), one obtains

$$
\begin{array}{r}
R_{0} C_{0} \frac{d z}{d t}=-\frac{R_{0}}{R_{x}} x-\frac{R_{0}}{R_{y}} y-\frac{R_{0}}{R_{z}} z+\frac{R_{0}\left(R i_{h}(x)\right)}{R} \\
+\frac{0.9 R_{0}\left(R i_{h}(y)\right)}{R}+\frac{0.825 R_{0}\left(R i_{h}(z)\right)}{R}
\end{array}
$$

where $R_{0}=0.8 \mathrm{k} \Omega, R_{x}=1 \mathrm{k} \Omega, R_{y}=1.11 \mathrm{k} \Omega$, $R_{z}=1.21 \mathrm{k} \Omega$, and $R=1 \mathrm{k} \Omega$. Let $a=R_{0} / R_{x}=R_{0} / R=0.8$, $b=R_{0} / R_{y}=0.9 R_{0} / R=0.72, c=R_{0} / R_{z}=$ $0.825 R_{0} / R=0.66, V_{h}(x)=R i_{h}(x), V_{h}(y)=R i_{h}(y)$, 


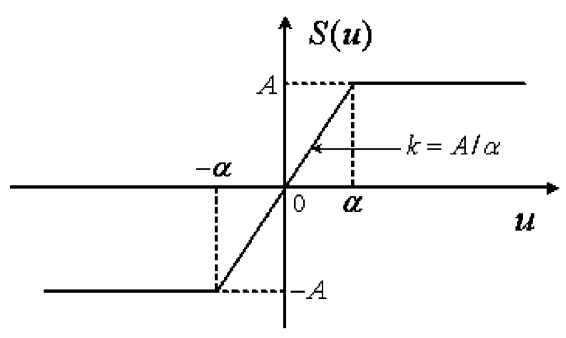

(a)

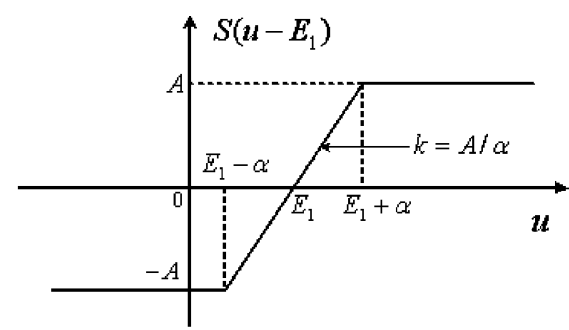

(b)

Fig. 9. (a) Voltage saturated function $S(u)$. (b) Delayed voltage saturated function $S\left(u-E_{1}\right)$.

and $V_{h}(z)=R i_{h}(z)$. The units of $V_{h}(x), V_{h}(y)$, and $V_{h}(z)$ are in volts.

Considering the time-scale transformation $t=R_{0} C_{0} \tau$, from (25), one has

$$
\frac{d z}{d \tau}=-a x-b y-c z+a V_{h}(x)+b V_{h}(y)+c V_{h}(z) \text {. }
$$

According to the operational amplifiers $O P_{3}$ and $O P_{6}$ shown in Fig. 3, one obtains

$$
\left\{\begin{array}{l}
\frac{d x}{d \tau}=y-\frac{V_{S}}{R_{d}} R i_{h}(y) \\
\frac{d y}{d \tau}=z-\frac{V_{S}}{R_{e}} R i_{h}(z)
\end{array}\right.
$$

where $V_{S}=7.5 \mathrm{~V}$ and $R_{d}=R_{e}=7.5 \mathrm{k} \Omega$. Thus, one has $\left(V_{S} / R_{d}\right) R i_{h}(y)=R i_{h}(y)=V_{h}(y)$ and $\left(V_{S} / R_{e}\right) R i_{h}(z)=$ $R i_{h}(z)=V_{h}(z)$. From (26)-(27), one obtains the normal state equation for 3-D hysteresis multiscroll chaotic attractors as follows:

$$
\left\{\begin{array}{l}
\frac{d x}{d \tau}=y-V_{h}(y) \\
\frac{d y}{d \tau}=z-V_{h}(z) \\
\frac{d z}{d \tau}=-a x-b y-c z+a V_{h}(x)+b V_{h}(y)+c V_{h}(z) .
\end{array}\right.
$$

Since (1) with controller (6) is the vector form of (28), we have thus derived the dynamic equation of the 3-D hysteresis multiscroll chaotic attractors.

\section{Design of Multidirectional SATURATED MulTisCROLl CiRCUITS}

Based on the voltage saturated function in this section, a simple block circuit diagram is described for hardware implementation of multidirectional saturated multiscroll chaotic attractors, including 1-D $n$-scroll, 2-D $n \times m$-grid scroll, and 3 -D $n \times m \times l$-grid scroll chaotic attractors.

\section{A. Voltage and Current Saturated Functions}

A voltage saturated function is described by

$$
S(u)=\frac{A}{2 \alpha}(|u+\alpha|-|u-\alpha|)= \begin{cases}-A, & u<-\alpha \\ \frac{A}{\alpha} u, & -\alpha \leq u \leq \alpha \\ A, & u>\alpha\end{cases}
$$

where $u \in\{x, y, z\}, \pm A$ are the values of saturated voltages, $\pm \alpha$ are the switching points, and $k=A / \alpha$ is the slope. Fig. 9(a) shows the above voltage saturated function $S(u)$.

Based on the voltage saturated function $S(u)$, one can further define the current saturated function as follows:

$$
i_{S}(u)=\frac{S(u)}{R_{C}}
$$

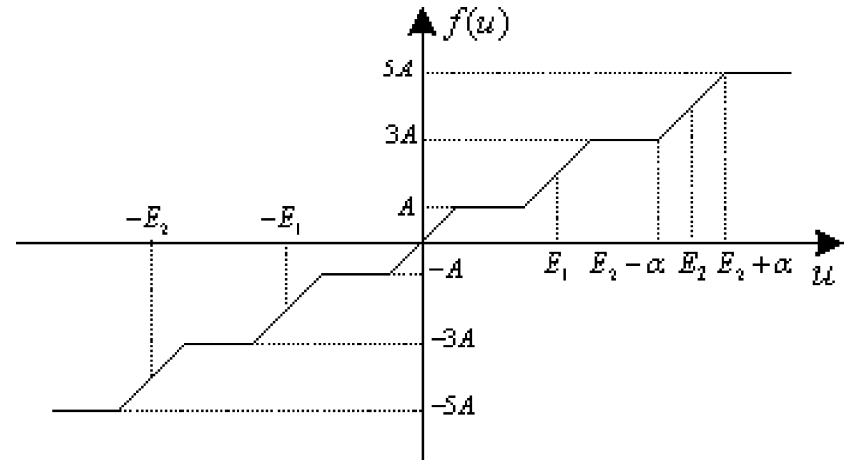

Fig. 10. Saturated function series $f(u)$.

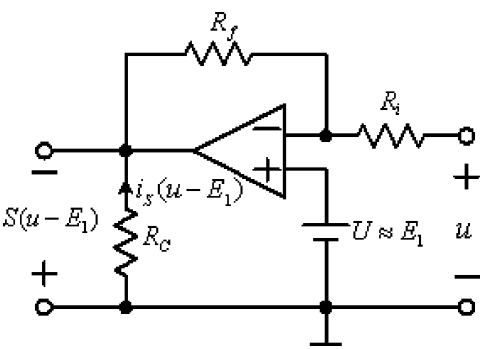

Fig. 11. Circuit cell for delayed voltage and current saturated functions. When $E_{1}=0$, that is, removing comparing voltage $U$, it also shows the circuit cell for voltage and current saturated functions.

where $R_{C}$ is a voltage-current conversion resistor. One can easily realize the transformation between the voltage saturated function (29) and the current saturated function (30) via the voltage-current conversion resistor $R_{C}$.

A delayed voltage saturated function is described by

$$
\begin{aligned}
S\left(u-E_{1}\right) & =\frac{A}{2 \alpha}\left(\left|u-E_{1}+\alpha\right|-\left|u-E_{1}-\alpha\right|\right) \\
& = \begin{cases}-A, & u<E_{1}-\alpha \\
\frac{A}{\alpha} u, & E_{1}-\alpha \leq u \leq E_{1}+\alpha \\
A, & u>E_{1}+\alpha\end{cases}
\end{aligned}
$$

where $u \in\{x, y, z\}, \pm A$ are the values of saturated voltages, $E_{1}$ is the delayed voltage, $E_{1} \pm \alpha$ are the switching points, and $k=A / \alpha$ is the slope. Fig. 9(b) shows the above delayed voltage saturated function $S\left(u-E_{1}\right)$.

Similarly, the delayed current saturated function is defined as follows:

$$
i_{S}\left(u-E_{1}\right)=\frac{S\left(u-E_{1}\right)}{R_{C}} .
$$




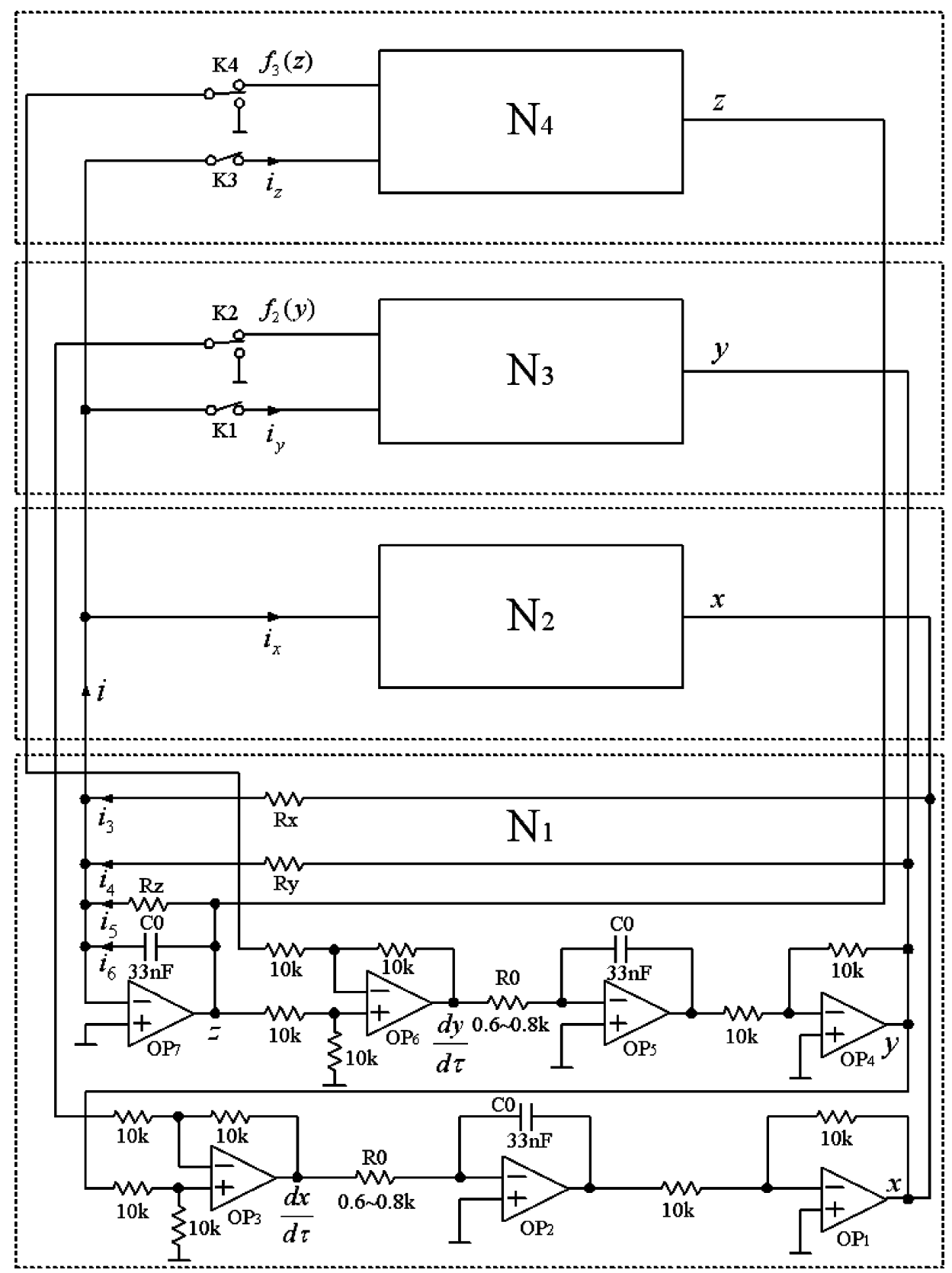

Fig. 12. Block circuit diagram of multidirectional saturated multiscroll chaotic circuits.

According to (29) and (31), the voltage saturated function series is described by

$$
\begin{aligned}
f(u)= & S(u)+\sum_{i=1}^{N} S\left(u-E_{i}\right)+\sum_{i=1}^{N} S\left(u+E_{i}\right)=\frac{A}{2 \alpha}(|u+\alpha|-|u-\alpha|) \\
& +\sum_{i=1}^{N} \frac{A}{2 \alpha}\left(\left|u-E_{i}+\alpha\right|-\left|u-E_{i}-\alpha\right|\right) \\
& +\sum_{i=1}^{N} \frac{A}{2 \alpha}\left(\left|u+E_{i}+\alpha\right|-\left|u+E_{i}-\alpha\right|\right) .
\end{aligned}
$$

Based on the voltage saturated function series $f(u)$, the current saturated function series is defined as follows:

$i(u)=\frac{f(u)}{R_{C}}=\frac{S(u)}{R_{C}}+\frac{1}{R_{C}} \sum_{i=1}^{N} S\left(u-E_{i}\right)+\frac{1}{R_{C}} \sum_{i=1}^{N} S\left(u+E_{i}\right)$.
Fig. 10 shows the voltage saturated function series with $N=2$ in (33).

\section{B. Circuit Realization for Voltage and Current Saturated Functions}

In this subsection, two circuit cells are constructed for realization of the voltage and the delayed voltage saturated functions, respectively.

Fig. 11 displays the circuit cell for realizing the delayed voltage and the delayed current saturated functions. Suppose that the comparing voltage in Fig. 11 is $U=E_{1} /\left(1+R_{i} / R_{f}\right)$. Then, the circuit cell can export the delayed voltage saturated function $S\left(u-E_{1}\right)$, as shown in Fig. 9(b), and the delayed current saturated function $i_{S}\left(u-E_{1}\right)=S\left(u-E_{1}\right) / R_{C}$. In Fig. 11, the operational amplifier is TL082; the voltages of the positive and negative electrical sources are $\pm E= \pm 15 \mathrm{~V}$; the 


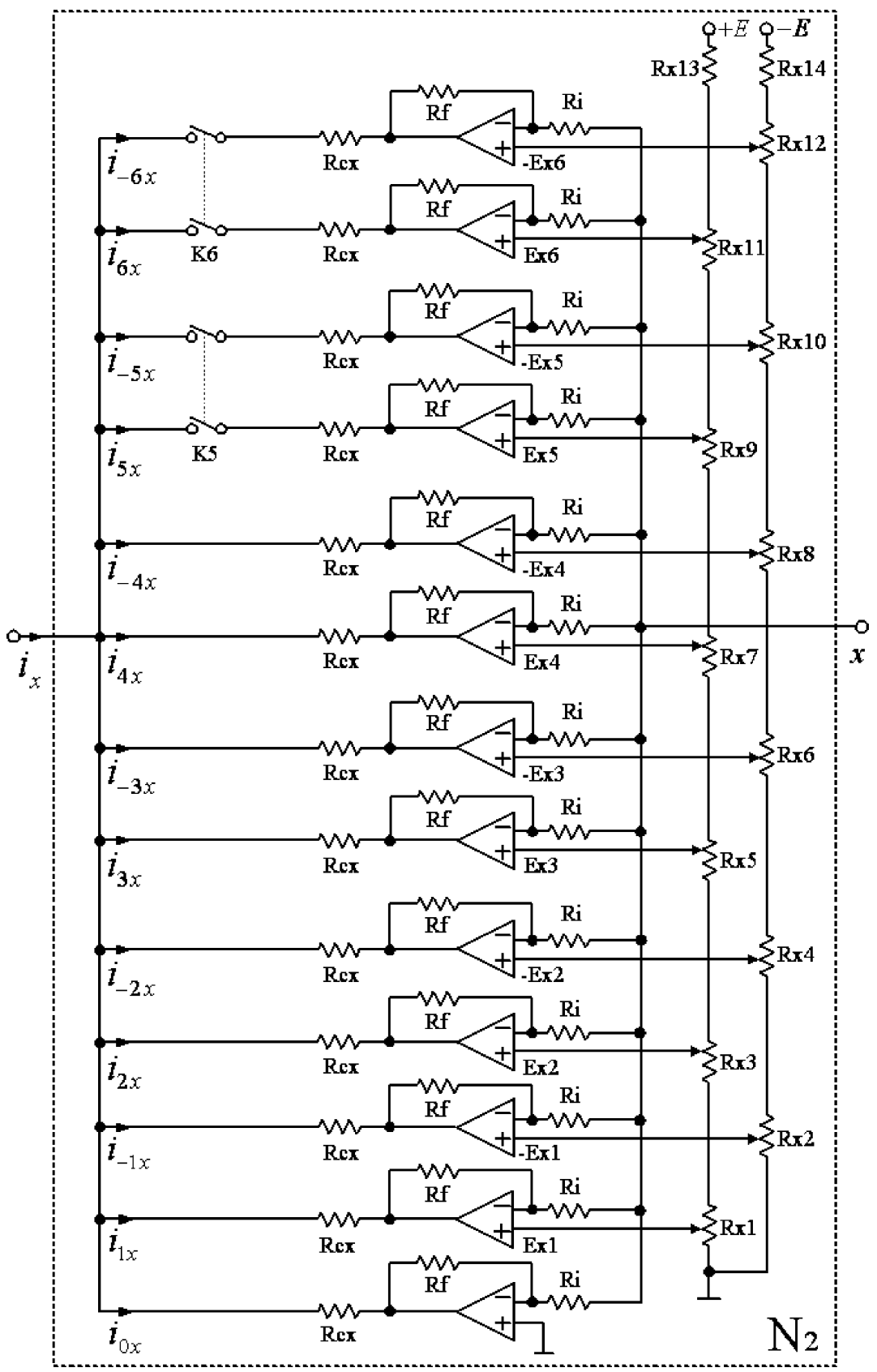

Fig. 13. Subcircuit diagram for generator $N_{2}$ of current saturated function series $i_{x}(x)$.

outputs of the saturated voltages are $\pm A= \pm\left|V_{\text {sat }}\right|= \pm 13.5 \mathrm{~V}$, the switching points are $E_{1} \pm \alpha=E_{1} \pm\left(R_{i}\left|V_{\text {sat }}\right| / R_{f}\right)$; the slope is $k=A / \alpha$; and the delayed voltage is $E_{1}$.

When $E_{1}=0$, that is, removing comparing voltage $U$ in Fig. 11, $S\left(u-E_{1}\right)=S(u), i_{S}\left(u-E_{1}\right)=i_{S}(u)$ and Fig. 11 also shows the circuit cell for realizing the voltage and the current saturated functions, which can export the voltage saturated function $S(u)$ as shown in Fig. 9(a) and the current saturated function $i_{S}(u)=S(u) / R_{C}$.

Here, assume that the ratio of resistor is $R_{f} / R_{i}=500$. Then, the comparing voltage in Fig. 11 is $U=E_{1} /\left(1+R_{i} / R_{f}\right) \approx$ $E_{1}$. In this case, the comparing voltage and the delayed voltage are almost same; that is, $U \approx E_{1}$. Based on this assumption, one can construct the more complex saturated function series circuit by using the saturated function circuit cell and the delayed sat- urated function circuit cell shown in Fig. 11 with different comparing voltages $U(U=0$ and $U \neq 0)$.

\section{Multdirectional Saturated Multiscroll Circuits and Experimental Observations}

In the following, the two basic circuit cells shown in Fig. 11 are used to construct the circuit for realizing the voltage saturated function series (33).

The designed circuit diagram for generating multidirectional saturated multiscroll chaotic attractors is shown in Figs. 12-14. The main block diagram Fig. 12 includes five different function parts: $N 1, N 2, N 3, N 4$, and the switch sets. More precisely, $N 1$ consists of three integrators, two inverter amplifiers and two subtractor amplifiers; $N 2$ consists of the generator for the $x$-directional current saturated function series $i_{x}(x)$ and the gener- 


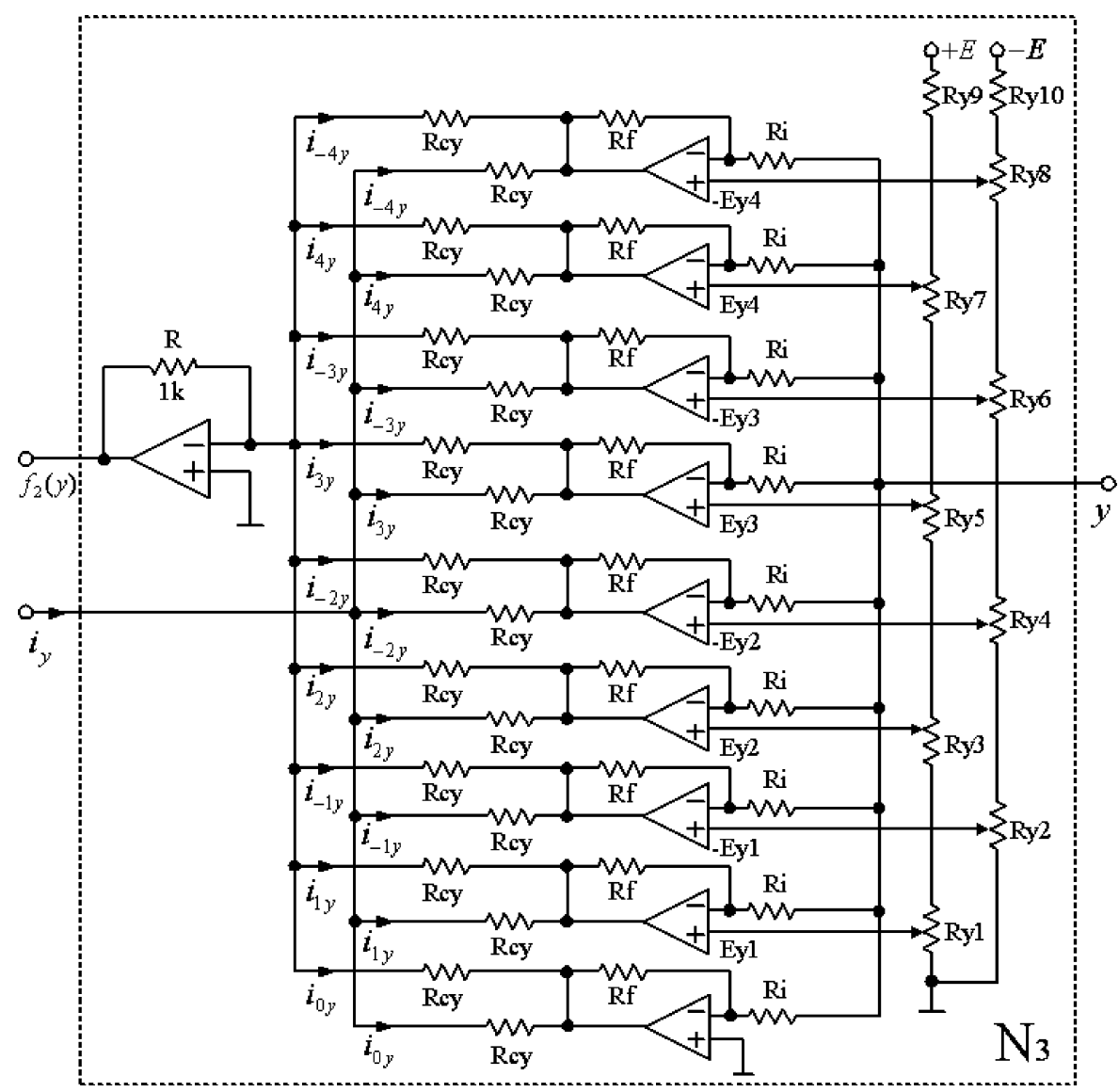

Fig. 14. Subcircuit diagram for generator $N_{3}$ of current saturated function series $i_{y}(y)$ and voltage saturated function series $f_{2}(y)$. After replacing all $y$ by $z$, $f_{2}(y)$ by $f_{3}(z)$, and $N_{3}$ by $N_{4}$, it also shows the subcircuit diagram for generator $N_{4}$ of current saturated function series $i_{z}(z)$ and voltage saturated function series $f_{3}(z)$.

ator for the $x$-directional voltage saturated function series $f_{1}(x)$, as shown in Fig. 13; $N 3$ consists of the generator for the $y$-directional current saturated function series $i_{y}(y)$ and the generator for the $y$-directional voltage saturated function series $f_{2}(y)$, as shown in Fig. 14; $N 4$ consists of the generator for the $z$-directional current saturated function series $i_{z}(z)$ and the generator for the $z$-directional voltage saturated function series $f_{3}(z)$, as shown in Fig. 14 with replacing all $y$ by $z, f_{2}(y)$ by $f_{3}(z)$, and $N_{3}$ by $N_{4}$. Switches $K 1, K 2, K 3, K 4$ shown in Fig. 12 are used to control the number of directions of the multiscroll chaotic attractors. When $K 1, K 3$ are switched off and $K 2, K 4$ are connected to the ground, the circuit generates 1-D $n$-scroll chaotic attractors; when $K 1, K 2$ are switched on, $K 3$ is switched off, and $K 4$ is connected to the ground, the circuit creates 2-D $n \times m$-grid scroll chaotic attractors; when $K 1, K 2, K 3, K 4$ are switched on, the circuit generates 3-D $n \times m \times l$-grid scroll chaotic attractors. Moreover, the linkage switches $K 5, K 6$ shown in Fig. 13 are used to control the number of scrolls in the $x$-direction. That is, when $K 5, K 6$ are switched off, the circuit generates 10 scrolls in the $x$-direction; when $K 5$ is switched on and $K 6$ is switched off, the circuit creates 12 scrolls in the $x$-direction; when $K 5, K 6$ are switched on, the circuit generates 14 scrolls in the $x$-direction.

Furthermore, all operational amplifiers shown in Figs. 12-14 are TL082, the supply voltages of the positive and negative electrical sources are $\pm E= \pm 15 \mathrm{~V}$, and the output saturated voltages of the operational amplifiers are $\left|V_{\text {sat }}\right|=13.5 \mathrm{~V}$. On the other hand, for convenient adjustment and higher precision, all resistors are precisely adjustable resistors or potentiometers. Here, $R_{c x}, R_{c y}, R_{c z}$ are the voltage-current conversion resistors. $\alpha=R_{i}\left|V_{\text {sat }}\right| / R_{f}$ is determined by resistors $R_{i}, R_{f}$ and the output saturated voltage $\left|V_{\text {sat }}\right|$. And, the comparing voltages $\pm E_{x i}(1 \leq i \leq 6)$ in Fig. $13, \pm E_{y i}(1 \leq i \leq 4)$ in Fig. 14, and $\pm E_{z i}(1 \leq i \leq 4)$ in Fig. 14 with replacing all $y$ by $z, f_{2}(y)$ by $f_{3}(z)$, and $N_{3}$ by $N_{4}$, are determined by $R_{x 1} \sim R_{x 14}$ in Fig. 13, $R_{y 1} \sim R_{y 10}$ in Fig. 14 , and $R_{z 1} \sim R_{z 10}$ in Fig. 14 with replacing all $y$ by $z, f_{2}(y)$ by $f_{3}(z)$, and $N_{3}$ by $N_{4}$, respectively. By adjusting the resistors $R_{x 1} \sim R_{x 14}, R_{y 1} \sim R_{y 10}$, and $R_{z 1} \sim R_{z 10}$, one can control the above corresponding comparing voltages, respectively. Thus, one can adjust the delayed voltages to generate the desired saturated function series in implementation. Note that $1 / R_{0} C_{0}$ is the integral constant of the integrators in $N 1$, which is also the transformation factor of the time-scale.

According to the main block circuit diagram Fig. 12, when $K 1, K 3$ are switched off and $K 2, K 4$ are connected to the ground, the circuitry shown in Fig. 12 can generate 1-D 10, 12, 14-scroll chaotic attractors by switching the linkage switches $K 5, K 6$ in Fig. 13. More precisely, when $K 5, K 6$ are switched off, the circuitry can create a 10 -scroll attractor in the $x$-direction; when $K 5$ is switched on and $K 6$ is switched off, the circuitry can generate a 12 -scroll attractor in the $x$-direction; when $K 5, K 6$ are switched on, the circuitry can create a 14-scroll attractor in the 
TABLE III

CiRCUIT PARAMETERS FOR THE REALIZATION OF 1-D SATURATED $N$-SCROLL CHAOTIC ATTRACTORS

\begin{tabular}{c|c|c|c|c|c}
\hline$R_{i}$ & $1 k \Omega$ & $R_{z}$ & $1 k \Omega$ & $\pm E_{x 3}$ & $\pm 3 V$ \\
\hline$R_{f}$ & $500 k \Omega$ & $R$ & $1 k \Omega$ & $\pm E_{x 4}$ & $\pm 4 V$ \\
\hline$R_{c x}$ & $27 k \Omega$ & $R_{0}$ & $0.6 \sim 0.8 k \Omega$ & $\pm E_{x 5}$ & $\pm 5 V$ \\
\hline$R_{x}$ & $1 k \Omega$ & $\pm E_{x 1}$ & $\pm 1 V$ & $\pm E_{x 6}$ & $\pm 6 V$ \\
\hline$R_{y}$ & $1 k \Omega$ & $\pm E_{x 2}$ & $\pm 2 V$ & $\left|V_{\text {sat }}\right|$ & $13.5 V$ \\
\hline
\end{tabular}

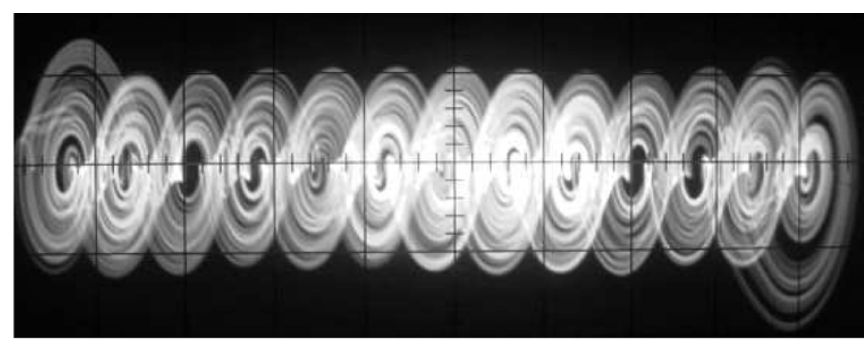

(a)

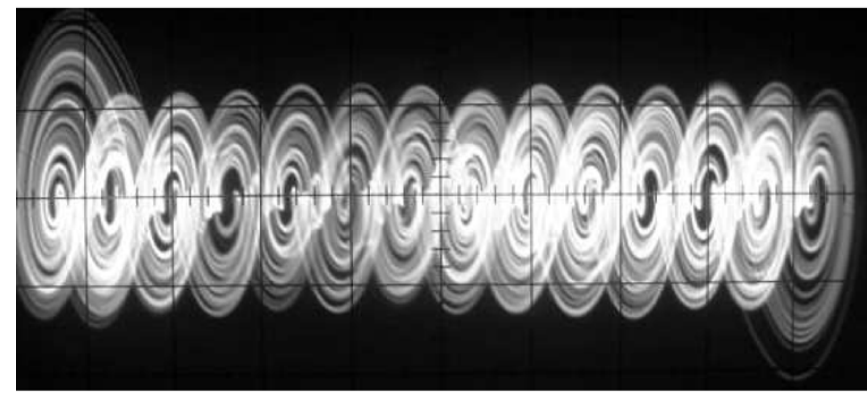

(b)

Fig. 15. Experimental observations of 1-D saturated multiscroll chaotic attractors in the $x-y$ plane. From up to down: (a) 13-scroll, where $x=1.3 \mathrm{~V} / \mathrm{div}, y=0.4 \mathrm{~V} /$ div. (b) 14 -scroll, where $x=1.4 \mathrm{~V} / \mathrm{div}$, $y=0.4 \mathrm{~V} /$ div .

$x$-direction. Here, all required circuit parameters are given in Table III. Note that the subcircuits $N 1, N 2$ are at work in this case, but the subcircuits $N 3, N 4$ are not at work.

Fig. 15 shows the experimental observations of 1-D 13, 14-scroll chaotic attractors in the $x-y$ plane. Here, the observed 13 -scroll chaotic attractor is verified by slightly readjusting the subcircuit $N 2$ in Fig. 13, as follows: (1) $K 5, K 6$ are switched on; (2) the subcurrent $i_{0 x}$ is switched off; (3) readjust the resistors $R_{x 1} \sim R_{x 4}$ to make the comparing voltages satisfying $\pm E_{x 1}= \pm 0.5 \mathrm{~V}, \pm E_{x 2}= \pm 1.5 \mathrm{~V}, \pm E_{x 3}= \pm 2.5 \mathrm{~V}$, $\pm E_{x 4}= \pm 3.5 \mathrm{~V}, \pm E_{x 5}= \pm 4.5 \mathrm{~V}$, and $\pm E_{x 6}= \pm 5.5 \mathrm{~V}$. It should be pointed out that all circuit parameters have to be readjusted for realizing attractors with an odd number of scrolls, since there always exist errors in electronic devices.

From Fig. 12, when $K 1, K 2$ are switched on, $K 2$ is switched off, and $K 4$ is connected to the ground, the circuitry creates 2-D $10,12,14 \times 10$-grid scroll chaotic attractors by switching the linkage switches $K 5, K 6$ in Fig. 13. That is, when $K 5, K 6$ are switched off, the circuitry generates a 2-D $10 \times 10$-grid scroll attractor in $x-y$ plane; when $K 5$ is switched on and $K 6$ is switched off, the circuitry creates a 2-D $12 \times 10$-grid scroll attractor in $x-y$ plane; when $K 5, K 6$ are switched on, the circuitry generates a 2-D $14 \times 10$-grid scroll attractor in $x-y$ plane. Here, all
TABLE IV

Circuit Parameters FOR THE REALIZATION OF 2-D $n \times m$-GRID SCROLL CHAOTIC ATTRACTORS

\begin{tabular}{c|c|c|c|c|c}
\hline$R_{i}$ & $1 k \Omega$ & $R$ & $1 k \Omega$ & $\pm E_{y 3}$ & $\pm 3 V$ \\
\hline$R_{f}$ & $500 k \Omega$ & $R_{0}$ & $0.6 \sim 0.8 k \Omega$ & $\pm E_{x 4}$ & $\pm 4 V$ \\
\hline$R_{c x}$ & $27 k \Omega$ & $\pm E_{x 1}$ & $\pm 1 V$ & $\pm E_{y 4}$ & $\pm 4 V$ \\
\hline$R_{c y}$ & $27 k \Omega$ & $\pm E_{y 1}$ & $\pm 1 V$ & $\pm E_{x 5}$ & $\pm 5 V$ \\
\hline$R_{x}$ & $1 k \Omega$ & $\pm E_{x 2}$ & $\pm 2 V$ & $\pm E_{x 6}$ & $\pm 6 V$ \\
\hline$R_{y}$ & $1 k \Omega$ & $\pm E_{y 2}$ & $\pm 2 V$ & $\left|V_{s a t}\right|$ & $13.5 V$ \\
\hline$R_{z}$ & $1 k \Omega$ & $\pm E_{x 3}$ & $\pm 3 V$ & & \\
\hline
\end{tabular}

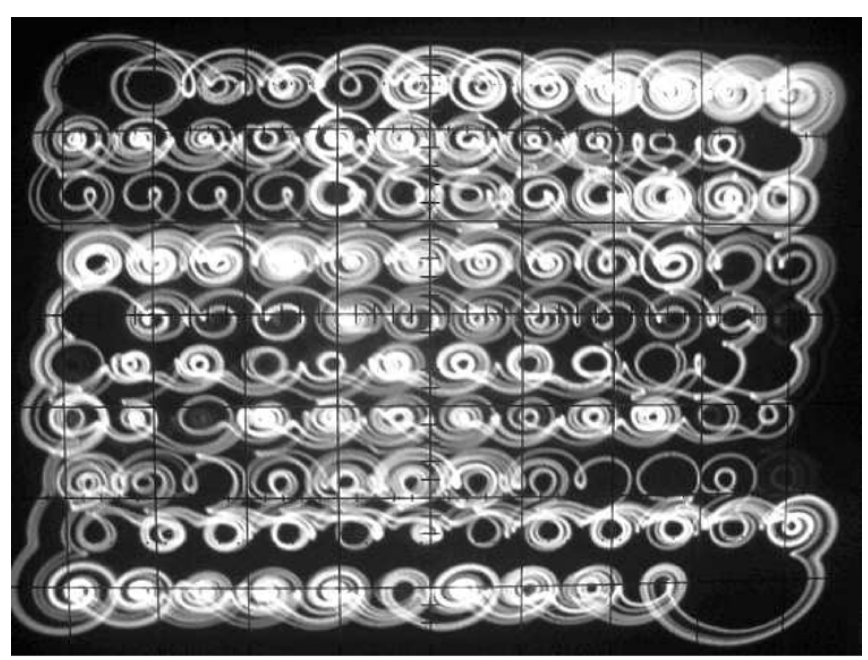

(a)

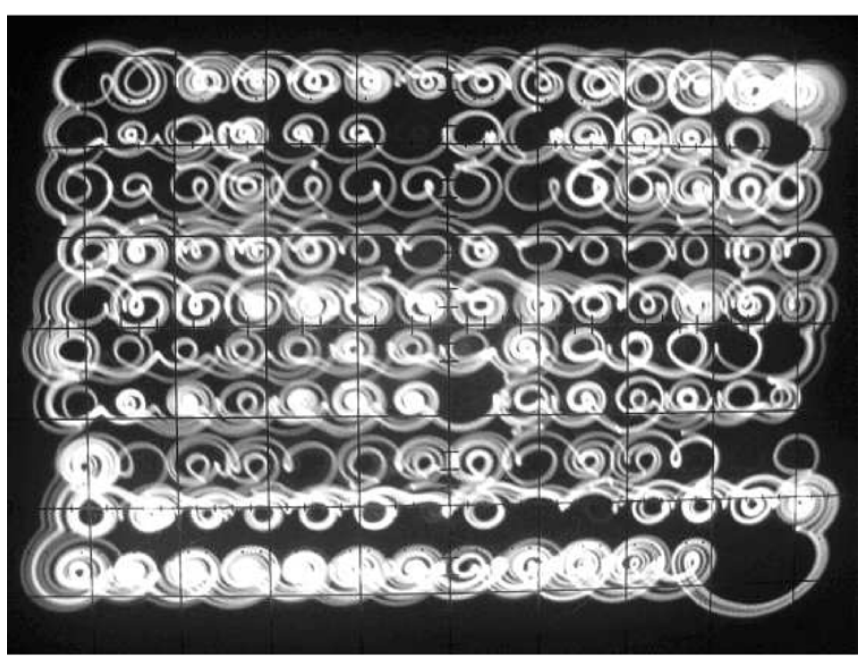

(b)

Fig. 16. Experimental observations of 2-D saturated multiscroll chaotic attractors in the $x-y$ plane. From up to down: (a) $12 \times 10$-grid scroll, where $x=1.2 \mathrm{~V} / \operatorname{div}, y=1.6 \mathrm{~V} /$ div. (b) $14 \times 10$-grid scroll, where $x=1.4 \mathrm{~V} / \mathrm{div}, y=1.6 \mathrm{~V} / \mathrm{div}$.

required circuit parameters are given in Table IV. Note that the subcircuits $N 1, N 2, N 3$ are at work in this case, but the subcircuits $N 4$ is not at work. Fig. 16 shows the experimental observations of 2-D 12, $14 \times 10$-grid scroll chaotic attractors in the $x$ $-y$ plane.When $K 1, K 2, K 3, K 4$ in Fig. 12 are switched on and $K 5, K 6$ in Fig. 13 are switched off, the circuitry generates a 3-D $10 \times 10 \times 10$-grid scroll chaotic attractor. Here, all required circuit parameters are given in Table V. Note that all the subcircuits 
TABLE V

CIRCUIT PARAMETERS FOR THE REALIZATION OF 3 -D $n \times m \times l$-GRID SCROLL CHAOTIC ATTRACTORS

\begin{tabular}{c|c|c|c|c|c}
\hline$R_{i}$ & $1 k \Omega$ & $R_{0}$ & $0.6 \sim 0.8 k \Omega$ & $\pm E_{y 2}$ & $\pm 1.0 \mathrm{~V}$ \\
\hline$R_{f}$ & $500 k \Omega$ & $\pm E_{x 1}$ & $\pm 1 V$ & $\pm E_{z 2}$ & $\pm 1.0 \mathrm{~V}$ \\
\hline$R_{c x}$ & $27 k \Omega$ & $\pm E_{x 2}$ & $\pm 2 \mathrm{~V}$ & $\pm E_{y 3}$ & $\pm 1.5 \mathrm{~V}$ \\
\hline$R_{c y}$ & $54 k \Omega$ & $\pm E_{x 3}$ & $\pm 3 V$ & $\pm E_{z 3}$ & $\pm 1.5 \mathrm{~V}$ \\
\hline$R_{c z}$ & $54 k \Omega$ & $\pm E_{x 4}$ & $\pm 4 V$ & $\pm E_{y 4}$ & $\pm 2.0 \mathrm{~V}$ \\
\hline$R_{x}$ & $1 k \Omega$ & $\pm E_{x 5}$ & $\pm 5 V$ & $\pm E_{z 4}$ & $\pm 2.0 \mathrm{~V}$ \\
\hline$R_{y}$ & $1 k \Omega$ & $\pm E_{x 6}$ & $\pm 6 V$ & $\left|V_{s a t}\right|$ & $13.5 \mathrm{~V}$ \\
\hline$R_{z}$ & $1 k \Omega$ & $\pm E_{y 1}$ & $\pm 0.5 V$ & & \\
\hline$R$ & $1 k \Omega$ & $\pm E_{z 1}$ & $\pm 0.5 \mathrm{~V}$ & & \\
\hline
\end{tabular}

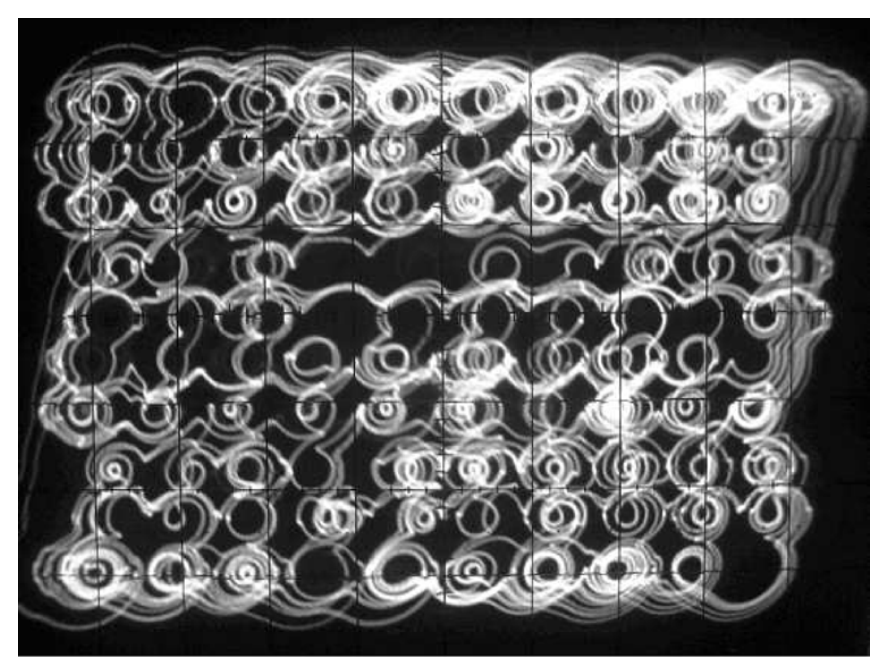

(a)

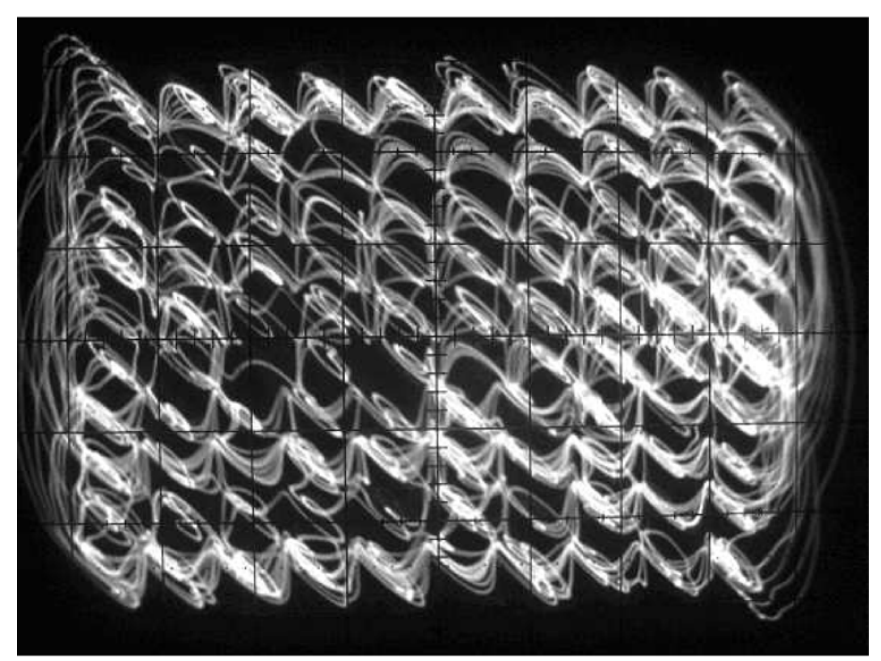

(b)

Fig. 17. Experimental observations of 3 -D saturated $10 \times 10 \times 10$-grid scroll chaotic attractors. From up to down: (a) In $x-y$ plane, where $x=1.0 \mathrm{~V} / \mathrm{div}$, $y=0.8 \mathrm{~V} /$ div. (b) In $x-z$ plane, where $x=1.0 \mathrm{~V} / \mathrm{div}, z=0.8 \mathrm{~V} / \mathrm{div}$.

$N 1, N 2, N 3, N 4$ are at work in this case. Fig. 17 shows the experimental observations of a 3 -D $10 \times 10 \times 10$-grid scroll chaotic attractor in the $x-y$ and $x-z$ planes, respectively.

\section{Theoretical Analysis of Multdirectional Saturated Multiscroll Circuits}

Here, the designed multidirectional saturated multiscroll circuits are further investigated; their dynamic equations are rigorously derived from the circuit diagrams Figs. 12-14. In the following, we only consider the $3-\mathrm{D}$ setting since $1-\mathrm{D}$ and $2-\mathrm{D}$ settings are special cases of 3-D setting.

According to the subcircuit N2 shown in Fig. 13, the total current saturated function series $i_{x}(x)$ in $x$-direction is given by

$$
\begin{aligned}
i_{x}(x)= & i_{0 x}+\sum_{n=1}^{6} K_{n} i_{n x}+\sum_{n=1}^{6} K_{n} i_{-n x} \\
= & \frac{1}{R_{c x}} \frac{\left|V_{\text {sat }}\right|}{2 \alpha_{x}}\left(\left|x+\alpha_{x}\right|-\left|x-\alpha_{x}\right|\right) \\
& +\sum_{n=1}^{6} \frac{K_{n}}{R_{c x}} \frac{\left|V_{\text {sat }}\right|}{2 \alpha_{x}}\left(\left|x-E_{x n}+\alpha_{x}\right|-\left|x-E_{x n}-\alpha_{x}\right|\right) \\
& +\sum_{n=1}^{6} \frac{K_{n}}{R_{c x}} \frac{\left|V_{\text {sat }}\right|}{2 \alpha_{x}}\left(\left|x+E_{x n}+\alpha_{x}\right|-\left|x+E_{x n}-\alpha_{x}\right|\right)
\end{aligned}
$$

where $K_{n}=1(1 \leq n \leq 4), K_{5}, K_{6}$ are the normal switch functions corresponding to the status of the switches $K 5, K 6$ in Fig. 13, respectively, $\alpha_{x}=R_{i}\left|V_{\text {sat }}\right| / R_{f}$, and all above requisite circuit parameters are given in Table III.

From the subcircuit $N 3$ shown in Fig. 14, the total current saturated function series $i_{y}(y)$ in the $y$-direction is given by

$$
\begin{aligned}
i_{y}(y)= & i_{0 y}+\sum_{n=1}^{4} i_{n y}+\sum_{n=1}^{4} i_{-n y} \\
= & \frac{1}{R_{c y}} \frac{\left|V_{\text {sat }}\right|}{2 \alpha_{y}}\left(\left|y+\alpha_{y}\right|-\left|y-\alpha_{y}\right|\right) \\
& +\sum_{n=1}^{4} \frac{1}{R_{c y}} \frac{\left|V_{\text {sat }}\right|}{2 \alpha_{y}}\left(\left|y-E_{y n}+\alpha_{y}\right|-\left|y-E_{y n}-\alpha_{y}\right|\right) \\
& +\sum_{n=1}^{4} \frac{1}{R_{c y}} \frac{\left|V_{\text {sat }}\right|}{2 \alpha_{y}}\left(\left|y+E_{y n}+\alpha_{y}\right|-\left|y+E_{y n}-\alpha_{y}\right|\right)(36)
\end{aligned}
$$

where $\alpha_{x}=\alpha_{y}=R_{i}\left|V_{\text {sat }}\right| / R_{f}$ and all the above required circuit parameters are given in Table IV. Moreover, $f_{2}(y)=$ $R i_{y}(y)$ creates the voltage saturated function series in the $y$-direction.

According to the subcircuit N4 shown in Fig. 14 with replacing all $y$ by $z, f_{2}(y)$ by $f_{3}(z)$, and $N_{3}$ by $N_{4}$, the total current saturated function series $i_{z}(z)$ in the $z$-direction is given by

$$
\begin{aligned}
i_{z}(z)= & i_{0 z}+\sum_{n=1}^{4} i_{n z}+\sum_{n=1}^{4} i_{-n z} \\
= & \frac{1}{R_{c z}} \frac{\left|V_{\mathrm{sat}}\right|}{2 \alpha_{z}}\left(\left|z+\alpha_{z}\right|-\left|z-\alpha_{z}\right|\right) \\
& +\sum_{n=1}^{4} \frac{1}{R_{c z}} \frac{\left|V_{\mathrm{sat}}\right|}{2 \alpha_{z}}\left(\left|z-E_{z n}+\alpha_{z}\right|-\left|z-E_{z n}-\alpha_{z}\right|\right) \\
& +\sum_{n=1}^{4} \frac{1}{R_{c z}} \frac{\left|V_{\mathrm{sat}}\right|}{2 \alpha_{z}}\left(\left|z+E_{z n}+\alpha_{z}\right|-\left|z+E_{z n}-\alpha_{z}\right|\right)(37)
\end{aligned}
$$


where $\alpha_{x}=\alpha_{y}=\alpha_{z}=R_{i}\left|V_{\text {sat }}\right| / R_{f}$ and all the above required circuit parameters are given in Table V. Furthermore, $f_{3}(z)=R i_{z}(z)$ generates the voltage saturated function series in the $z$-direction. From (35), (36), and (37), the total current saturated function series is $i=i_{x}(x)+i_{y}(y)+i_{z}(z)$.

Therefore, according to subcircuit $N 1$, the circuit equation of the operational amplifier $O P 7$ is given by

$$
i_{3}+i_{4}+i_{5}+i_{6}=i
$$

where $i=i_{x}(x)+i_{y}(y)+i_{z}(z)$. Here, $i_{x}(x), i_{y}(y), i_{z}(z)$, and $i_{3}, i_{4}, i_{5}, i_{6}$ are, respectively, defined by (35)- (37), and

$$
\left\{\begin{array}{l}
i_{3}=\frac{x}{R_{x}} \\
i_{4}=\frac{y^{x}}{R_{y}} \\
i_{5}=\frac{z}{R_{z}} \\
i_{6}=C_{0} \frac{d z}{d t} .
\end{array}\right.
$$

In Fig. 12 , let $R_{0}=0.6 \sim 0.8 \mathrm{k} \Omega$. Multiplying $R_{0}$ to both sides of (38), and using (38) and (39), one has

$$
\begin{aligned}
R_{0} C_{0} \frac{d z}{d t} & =-\frac{R_{0}}{R_{x}} x-\frac{R_{0}}{R_{y}} y-\frac{R_{0}}{R_{z}} z \\
& +\frac{R_{0}}{R}\left(R i_{x}(x)\right)+\frac{R_{0}}{R}\left(R i_{y}(y)\right)+\frac{R_{0}}{R}\left(R i_{z}(z)\right)
\end{aligned}
$$

where $f_{1}(x)=R i_{x}(x), f_{2}(y)=R i_{y}(y)$, and $f_{3}(z)=R i_{z}(z)$ generate the voltage saturated function series in the $x, y, z$-directions, respectively. Considering the time-scale transformation $t=R_{0} C_{0} \tau$, from (40), one has

$$
\frac{d z}{d \tau}=-a x-a y-a z+a f_{1}(x)+a f_{2}(y)+a f_{3}(z) .
$$

From Table V, $a=0.6 \sim 0.8$. By changing resistor $R_{0}$, one can adjust the system parameter $a$.

Moreover, according to the operational amplifiers $O P_{3}$ and $O P_{6}$ of subcircuit $N 1$, shown in Fig. 12, one has

$$
\left\{\begin{array}{l}
\frac{d x}{d \tau}=y-f_{2}(y) \\
\frac{d y}{d \tau}=z-f_{3}(z) .
\end{array}\right.
$$

From (41)-(42), one attains the normal state equation of 3-D saturated multiscroll attractors as follows:

$$
\left\{\begin{array}{l}
\frac{d x}{d \tau}=y-f_{2}(y) \\
\frac{d y}{d \tau}=z-f_{3}(z) \\
\frac{d z}{d \tau}=-a x-a y-a z+a f_{1}(x)+a f_{2}(y)+a f_{3}(z) .
\end{array}\right.
$$

According to Table $\mathrm{V}$, one can get the system parameters of $f_{1}(x)$ as follows: saturated voltage $A=R\left|V_{\text {sat }}\right| / R_{c x}=0.5 \mathrm{~V}$, $\alpha_{x}=R_{i}\left|V_{\text {sat }}\right| / R_{f}=0.027 \mathrm{~V}$, slope $k=A / \alpha_{x}=18.5$, delayed voltages $\pm E_{x n}(1 \leq n \leq 6)$ defined by Table $\mathrm{V}$; the system parameters of $f_{z}(y)$ as follows: saturated voltage $A=$ $R\left|V_{\text {sat }}\right| / R_{c y}=0.25 \mathrm{~V}, \alpha_{y}=R_{i}\left|V_{\text {sat }}\right| / R_{f}=0.027 \mathrm{~V}$, slope $k=A / \alpha_{y}=9.26$, delayed voltages $\pm E_{y n}(1 \leq n \leq 4)$ given by Table V; the system parameters of $f_{3}(z)$ as follows: saturated voltage $A=R\left|V_{\text {sat }}\right| / R_{c z}=0.25 \mathrm{~V}, \alpha_{z}=R_{i}\left|V_{\text {sat }}\right| / R_{f}=$ $0.027 \mathrm{~V}$, slope $k=A / \alpha_{z}=9.26$, delayed voltages $\pm E_{z n}$ $(1 \leq n \leq 4)$ defined by Table V. Based on above parameters, one can deduce the detailed expressions of $f_{1}(x), f_{2}(y)$, and $f_{3}(z)$ in (43).

Remark 2: It is clear that there are several holes in Fig. 17(a). This is because the probabilities of appearance for the scrolls lying in the position of the holes are very low. Moreover, it indicates that the multidirectional multiscroll chaotic attractors become more and more unstable with the increase in the number of scrolls and the number of directions.

\section{CONCLUSION}

In this paper, a systematic methodology for circuit design has been proposed for experimental verification of multidirectional multiscroll chaotic attractors, including 1-D $n$-scroll, 2-D $n \times m$-grid scroll, and 3-D $n \times m \times l$-grid scroll chaotic attractors. More precisely, a simple block circuitry diagram has been designed for physically realizing 1-D $5 \sim 11$-scroll, 2-D $3 \times 5 \sim 11$-grid scroll, and 3-D $3 \times 3 \times 5 \sim 11$-grid scroll hysteresis chaotic attractors via simple switchings. Moreover, a novel block circuitry has been designed for experimentally verifying the saturated 1-D 10, 12, 14-scroll, 2-D 10, 12, $14 \times 10$-grid scroll, and 3-D $10 \times 10 \times 10$-grid scroll chaotic attractors via switching. It has been demonstrated that one can easily physically realize chaotic attractors with an odd number of scrolls by slightly modifying the corresponding voltage saturated function series of the circuitry, so as to generate for instance a 1-D 13-scroll chaotic attractor. This is the first time in the literature to report the experimental verification of 1-D 14-scroll, 2-D $14 \times 10$-grid scroll, and 3-D $10 \times 10 \times 10$-grid scroll chaotic attractors. The reported experiments have also shown that the realization of 3-D $n \times m \times l$-grid scroll with $n, m, l \geq 10$ chaotic attractors is much more difficult than that for 1-D $n$-scroll with $n \geq 10$ and 2-D $n \times m$-grid scroll with $n, m \geq 10$ chaotic attractors.

As can be seen throughout this paper, the proposed design idea and approach actually indicate a theoretical principle for hardware implementation of chaotic attractors with a multidirectional orientation and with a large number of scrolls. It is believed that this design methodology will provide a useful tool for many real-world engineering applications that require complex multiscroll chaotic attractors generated by simple and reliable electronic circuits.

\section{REFERENCES}

[1] G. Zhong, K. F. Man, and G. Chen, "A systematic approach to generating $n$-scroll attractors," Int. J. Bifurc. Chaos, vol. 12, no. 12, pp. 2907-2915, Dec. 2002.

[2] K. S. Tang, G. Q. Zhong, G. Chen, and K. F. Man, "Generation of $n$-scroll attractors via sine function," IEEE Trans. Circuits Syst. I, Fundam. Theory Appl., vol. 48, no. 11, pp. 1369-1372, Nov. 2001.

[3] J. Lü and G. Chen, "Multi-scroll Chaos generation: Theories, methods and applications,' Int. J. Bifurc. Chaos, vol. 16, no. 4, Apr. 2006, to be published.

[4] M. E. Yalcin, "Cellular neural networks, multi-scroll chaos and synchronization: theory, applications and implementations," Ph.D. dissertation, Katholieke Universiteit Leuven, Heverlee, Belgium, 2004.

[5] J. A. K. Suykens and J. Vandewalle, "Quasilinear approach to nonlinear systems and the design of $n$-double scroll $(n=1,2,3,4, \ldots)$," Proc. Inst. Elect. Eng., pt. G, vol. 138, no. 5, pp. 595-603, Oct. 1991.

[6] — "Generation of $n$-double scrolls $(n=1,2,3,4, \ldots)$," IEEE Trans. Circuits Syst. I, Fundam. Theory Appl., vol. 40, no. 11, pp. 861-867, Nov. 1993.

[7] J. A. K. Suykens and L. O. Chua, " $n$-double scroll hypercubes in 1-D CNNs," Int. J. Bifurc. Chaos, vol. 7, no. 8, pp. 1873-1885, Aug. 1997. 
[8] J. A. K. Suykens, A. Huang, and L. O. Chua, "A family of $n$-scroll attractors from a generalized Chua's circuit," Int. J. Electron. Commun., vol. 51, no. 3, pp. 131-138, 1997.

[9] M. E. Yalcin, S. Ozoguz, J. A. K. Suykens, and J. Vandewalle, " $n$-scroll Chaos generators: A simple circuit model," Electron. Lett., vol. 37, no. 3, pp. 147-148, Feb. 2001

[10] M. E. Yalcin, J. A. K. Suykens, J. Vandewalle, and S. Ozoguz, "Families of scroll grid attractors," Int. J. Bifurc. Chaos, vol. 12, no. 1, pp. 23-41, Jan. 2002.

[11] P. Arena, S. Baglio, L. Fortuna, and G. Manganaro, "Generation of $n$-double scrolls via cellular neural networks," Int. J. Circuit Theory Appl., vol. 24, no. 3, pp. 241-252, May/Jun. 1996.

[12] M. A. Aziz-Alaoui, "Differential equations with multispiral attractors," Int. J. Bifurc. Chaos, vol. 9, no. 6, pp. 1009-1039, Jun. 1999.

[13] M. E. Yalcin, J. A. K. Suykens, and J. Vandewalle, "Hyperchaotic $n$-scroll attractors," in Proc. IEEE Workshop on Nonlinear Dynamics of Electronic Systems (NDES 2000), Catania, Italy, May 18-20, 2000, pp. 25-28.

[14] S. Ozoguz, A. S. Elwakil, and K. N. Salama, " $n$-scroll Chaos generator using nonlinear transconductor," Electron. Lett., vol. 38, no. 14, pp. 685-686, Jul. 2002.

[15] J. Lü, T. Zhou, G. Chen, and X. Yang, "Generating Chaos with a switching piecewise-linear controller," Chaos, vol. 12, no. 2, pp. 344-349, Jun. 2002.

[16] J. Lü, X. Yu, and G. Chen, "Generating chaotic attractors with multiple merged basins of attraction: A switching piecewise-linear control approach," IEEE Trans. Circuits Syst. I, Fundam. Theory Appl., vol. 50, no. 2, pp. 198-207, Feb. 2003.

[17] R. W. Newcomb and N. El-Leithy, "Chaos generation using binary hysteresis," Circuit Syst., Signal Process., vol. 5, no. 3, pp. 321-341, 1986.

[18] T. Saito, "An approach toward higher dimensional hysteresis Chaos generators," IEEE Trans. Circuits Syst. I, Fundam. Theory Appl., vol. 37, no. 3, pp. 399-409, Mar. 1990.

[19] S. Nakagawa and T. Saito, "An RC OTA hysteresis Chaos generator," IEEE Trans. Circuits Syst. I, Fundam. Theory Appl., vol. 43, no. 12, pp. 1019-1021, Dec. 1996.

[20] M. Storace, M. Parodi, and D. Robatto, "A hysteresis-based chaotic circuit: Dynamics and applications," Int. J. Circuit Theor. Appl., vol. 27, no. 6, pp. 527-542, Nov./Dec. 1999.

[21] A. S. Elwakil and M. P. Kennedy, "Chaotic oscillators derived from Saito's double-screw hysteresis oscillator," IEICE Trans. Fundamentals, vol. E82-A, no. 9, pp. 1769-1775, Sep. 1999.

[22] - "Systematic realization of a class of hysteresis chaotic oscillators," Int. J. Circuit Theor. Appl., vol. 28, no. 4, pp. 319-334, Jul./Aug. 2000.

[23] P. Arena, S. Baglio, L. Fortuna, and G. Manganaro, "Hyperchaos from cellular neural networks," Electron. Lett., vol. 31, no. 4, pp. 250-251, Feb. 1995.

[24] J. E. Varrientos and E. Sánchez-Sinencio, "A 4-D chaotic oscillator based on a differential hysteresis comparator," IEEE Trans. Circuits Syst. I, Fundam. Theory Appl., vol. 45, no. 1, pp. 3-10, Jan. 1998.

[25] M. Kataoka and T. Saito, "A two-port VCCS chaotic oscillator and quad screw attractor," IEEE Trans. Circuits Syst. I, Fundam. Theory Appl., vol. 48, no. 2, pp. 221-225, Feb. 2001.

[26] J. Lü, X. Yu, and G. Chen, "Switching control for multi-scroll Chaos generation: An overview," in Proc. Phys. Contr., vol. 2, Saint Petersburg, Russia, Aug. 20-22, 2003, pp. 420-428.

[27] J. Lü, "Switching control: From simple rules to complex chaotic systems," J. Syst. Science and Complexity, vol. 16, no. 3, pp. 404-413, Jul. 2003.

[28] F. Han, X. Yu, Y. Wang, Y. Feng, and G. Chen, " $n$-scroll chaotic oscillators by second-order systems and double-hysteresis blocks," Electron. Lett., vol. 39, no. 23, pp. 1636-1638, Nov. 2003.

[29] F. Han, J. Lü, X. Yu, G. Chen, and Y. Feng, "Generating multi-scroll chaotic attractors via a linear second-order hysteresis system," Dynam. Continuous, Discr.Impulsive Syst. Series B: Appl. Alg., vol. 12, no. 1, pp. 95-110, Feb. 2005.

[30] J. Lü, F. Han, X. Yu, and G. Chen, "Generating 3-D multi-scroll chaotic attractors: A hysteresis series switching method," Automatica, vol. 40, no. 10, pp. 1677-1687, Oct. 2004.

[31] J. Lü, G. Chen, X. Yu, and H. Leung, "Design and analysis of multi-scroll chaotic attractors from saturated function series," IEEE Trans. Circuits Syst. I, Reg. Papers, vol. 51, no. 12, pp. 2476-2490, Dec. 2004.

[32] - "Generating multi-scroll chaotic attractors via switching control," in Proc. 5th Asian Control Conf., Melbourne, Australia, Jul. 20-23, 2004, pp. 1763-1771.
[33] S. M. Yu, J. Lü, H. Leung, and G. Chen, "Design and implementation of $n$-scroll chaotic attractors from a general jerk circuit," IEEE Trans. Circuits Syst. I, Reg. Papers, vol. 52, no. 7, pp. 1459-1477, Jul. 2005.

[34] - "N-scroll chaotic attractors from a general jerk circuit," in Proc. IEEE Int. Symp. Circuits and Systems (ISCAS'05), Kobe, Japan, May 23-26, 2005, pp. 1473-1476.

[35] S. M. Yu, S. S. Qiu, and Q. H. Lin, "New results of study on generating multiple-scroll chaotic attractors," Science in China Series F, vol. 46, no. 2, pp. 104-115, Feb. 2003.

[36] S. M. Yu, Z. G. Ma, S. S. Qiu, S. G. Peng, and Q. H. Lin, "Generation and synchronization of $n$-scroll chaotic and hyperchaotic attractors in fourth-order systems," Chinese Phys., vol. 13, no. 3, pp. 317-328, Mar. 2004.

[37] M. E. Yalcin, J. A. K. Suykens, and J. Vandewalle, "Experimental confirmation of 3- and 5-scroll attractors from a generalized Chua's circuit," IEEE Trans. Circuits Syst. I, Fundam. Theory Appl., vol. 47, no. 3, pp. 425-429, Mar. 2000.

[38] J. Lü, S. M. Yu, H. Leung, and G. Chen, "Experimental verification of 3-D hysteresis multi-scroll chaotic attractors," in Proc. IEEE Int. Symp. Circuits Systems (ISCAS'05), Kobe, Japan, May 23-26, 2005, pp. 3391-3394.

[39] T. Matsumoto, L. O. Chua, and K. Kobayashi, "Hyperchaos: Laboratory experiment and numerical confirmation," IEEE Trans. Circuits Syst., vol. CAS-33, no. 11, pp. 1143-1147, Nov. 1986.

[40] M. E. Yalcin, J. A. K. Suykens, and J. Vandewalle, "On the realization of $n$-scroll attractors," in Proc. IEEE Int. Symp. Circuits and Systems (ISCAS'99), vol. 5, Orlando, FL, May-Jun. 30-2, 1999, pp. 483-486.

[41] S. M. Yu, Q. H. Lin, and S. S. Qiu, "A family of multiple folded torus chaotic attractors" (in Chinese), Acta Physica Sinica, vol. 53, no. 7, pp. 2084-2088, Jul. 2004.

[42] A. S. Elwakil and M. P. Kennedy, "Construction of classes of circuitindependent chaotic oscillators using passive-only nonlinear devices," IEEE Trans. Circuits Syst. I, Fundam. Theory Appl., vol. 48, no. 3, pp. 289-307, Mar. 2001.

[43] P. Arena, S. Baglio, L. Fortuna, and G. Manganaro, "State controlled CNN: A new strategy for generating high complex dynamics," IEICE Trans. Fundamentals, vol. E79-A, no. 10, pp. 1647-1657, Oct. 1996.

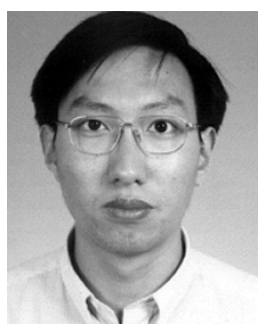

Jinhu Lü (M'03) received the B.Sc. degree in mathematics, the M.Sc. degree in applied mathematics, and the $\mathrm{Ph} . \mathrm{D}$ degree in applied mathematics, from Hubei Normal University, Wuhan University, and Chinese Academy of Sciences, Beijing, in 1997, 2000, and 2002, respectively.

Currently, he is a Visiting Fellow in the Department of Ecology and Evolutionary Biology, Princeton University, Princeton, NJ, and an Assistant Professor with the Institute of Systems Science, Academy of Mathematics and Systems Sciences, Chinese Academy of Sciences. He was a Postdoctoral Fellow with the Department of Electrical and Computer Engineering, University of Calgary, Calgary, Canada, from June 2004 to August 2005. He held several visiting positions in City University of Hong Kong, Hong Kong SAR, Royal Melbourne Institute of Technology University, Melbourne, Australia, and Santa Fe Institute, NM. He is the author of two research monographs and more than 60 research journal papers published in the fields of nonlinear circuits and systems, complex networks, and complex systems.

Dr. Lü served as a member of Technical Committee of several international conferences and is now serving as a member of the Technical Committee on Nonlinear Circuits and Systems of the IEEE Circuits and Systems Society. He is an Associate Editor of the Journal of Systems Science and Complexity. He received the Presidential Outstanding Research Award from the Chinese Academy of Sciences in 2002 and the 100 National Best Ph.D. Theses Award from the Office of Academic Degrees Committee of the State Council and the Ministry of Education of China in 2004. 


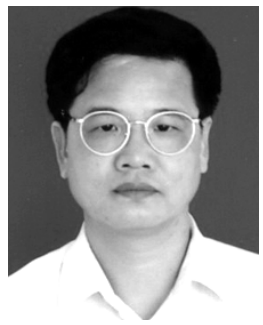

Simin Yu received the B.Sc. degree in electronics from Yunnan University, Kunming, China, in 1983, the M.E. degree in radio communication engineering, and the Ph.D. degree in nonlinear circuits and systems both from the South China University of Technology, Guangzhou, in 1996 and 2001, respectively.

Currently, he is a full Professor with the College of Automation, Guangdong University of Technology, Guangzhou. His research interests include the design and analysis of nonlinear circuits, computer simulations, and wireless communications. He has published more than 30 research journal papers in the fields of chaos in electronic circuits and chaotic communications.

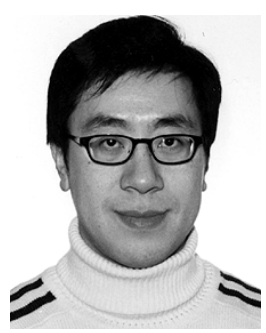

Henry Leung (M'90) received the Ph.D. degree in electrical and computer engineering from the McMaster University, Canada.

$\mathrm{He}$ is currently a Professor with the Department of Electrical and Computer Engineering, University of Calgary, Canada. Prior to that, he was with the Defence Research Establishment, Ottawa, Canada, where he was involved in the design of automated systems for air and maritime multisensor surveillance. His research interests include chaos, computational intelligence, data mining, nonlinear signal processing, multimedia, radar, sensor fusion, and wireless communications.

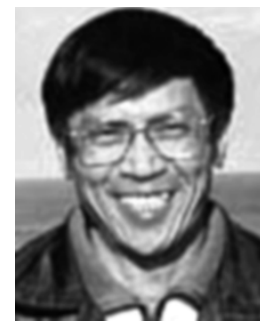

abstracts.

Prof. Chen served and is serving as Editor in various ranks for eight international journals including the IEEE TRANSACTIONS ON CIRCUITS AND SYSTEMS, as well as the IEEE Circuits and Systems Magazine, the IEEE TRANSACTIONS ON AUTOMATIC CONTROL, and the International Journal of Bifurcation and Chaos. He received the 1998 Harden-Simons Annual Prize for Outstanding Journal Paper from the American Society of Engineering Education, the 2001 IEEE M. Barry Carlton Best Annual Paper Award from the IEEE Aerospace and Electronic Systems Society, the 2002 Best Paper Award from the Institute of Information Theory and Automation, Academy of Sciences of the Czech Republic, and the 2005 IEEE Guillemin-Cauer Best Annual Paper Award from the IEEE Circuits and Systems Society. He is an Honorary Professor of the Central Queensland University, Australia, as well as Honorary Guest-Chair Professors of more than 10 universities in China. He was elected a Fellow of the IEEE for his fundamental contributions to the theory and applications of chaos control and bifurcation analysis 